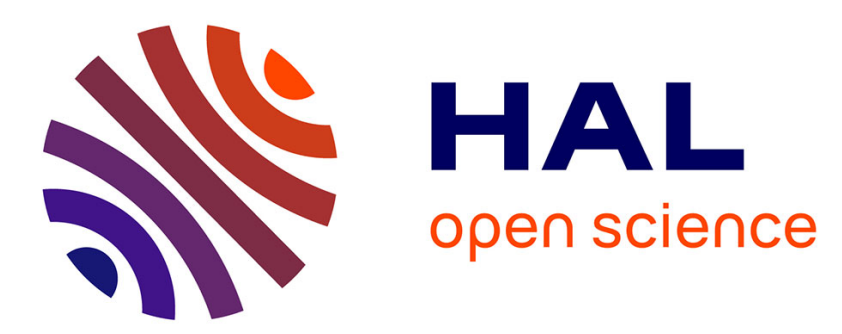

\title{
Dusty Infrared Galaxies: Sources of the Cosmic Infrared Background
}

\author{
Guilaine Lagache, Jean-Loup Puget, Herve Dole
}

\section{To cite this version:}

Guilaine Lagache, Jean-Loup Puget, Herve Dole. Dusty Infrared Galaxies: Sources of the Cosmic Infrared Background. 2005. hal-01840625

\section{HAL Id: hal-01840625 \\ https://hal-amu.archives-ouvertes.fr/hal-01840625}

Preprint submitted on 16 Jul 2018

HAL is a multi-disciplinary open access archive for the deposit and dissemination of scientific research documents, whether they are published or not. The documents may come from teaching and research institutions in France or abroad, or from public or private research centers.
L'archive ouverte pluridisciplinaire HAL, est destinée au dépôt et à la diffusion de documents scientifiques de niveau recherche, publiés ou non, émanant des établissements d'enseignement et de recherche français ou étrangers, des laboratoires publics ou privés. 
To Appear in Annual Reviews of Astronomy \& Astrophysics, 2005, volume 43

Preprint typeset using $\mathrm{L}_{\mathrm{A}} \mathrm{T}_{\mathrm{E}} \mathrm{X}$ style emulateapj v. 2/19/04

\title{
DUSTY INFRARED GALAXIES: SOURCES OF THE COSMIC INFRARED BACKGROUND \\ Guilaine Lagache, Jean-Loup Puget, Hervé Dole \\ Institut d'Astrophysique Spatiale, Bât 121, Université de Paris Sud 11, 91405 Orsay Cedex, France; e-mail: guilaine.lagache@ias.u-psud.fr, puget@ias.u-psud.fr, herve.dole@ias.u-psud.fr \\ To Appear in Annual Reviews of Astronomy 83 Astrophysics, 2005, volume 43
}

\begin{abstract}
The discovery of the Cosmic Infrared Background (CIB) in 1996, together with recent cosmological surveys from the mid-infrared to the millimeter have revolutionized our view of star formation at high redshifts. It has become clear, in the last decade, that a population of galaxies that radiate most of their power in the far-infrared (the so-called "infrared galaxies") contributes an important part of the whole galaxy build-up in the Universe. Since 1996, detailed (and often painful) investigations of the high-redshift infrared galaxies have resulted in the spectacular progress covered in this review. We outline the nature of the sources of the CIB including their star-formation rate, stellar and total mass, morphology, metallicity and clustering properties. We discuss their contribution to the stellar content of the Universe and their origin in the framework of the hierarchical growth of structures. We finally discuss open questions for a scenario of their evolution up to the present-day galaxies.

Subject headings: cosmology - evolution - luminosity function - starburst - star formation
\end{abstract}

\section{INTRODUCTION}

The Cosmic Infrared Background (CIB) can be defined as the part of the present radiation content of the Universe that is made essentially of the long wavelength output from all sources throughout the history of the Universe. The radiation content in the microwave part of the spectrum is dominated by the Cosmic Microwave Background ( $\mathrm{CMB}$ ) produced in the hot and dense phases of the universe. It dominates for frequencies below $800 \mathrm{GHz}$. Nevertheless the very different spectra of the CIB with respect to the CMB (both its purely Planckian part and its Compton distortion expected to be the dominant one at these frequencies) allow them to be separated very efficiently down to frequencies close to the peak of the CMB (150 $\mathrm{GHz}$ ). Furthermore in this frequency regime the CIB dominates the galactic emission in the lowest cirrus regions by a factor $\simeq 4$. The cosmic background due to sources (CMB excluded) presents two maxima: one in the optical, one in the far-infrared, with roughly equal brightness (in $\nu I_{\nu}$ ) and with a minimum around $5 \mu \mathrm{m}$. This minimum is created by the decrease of brightness of the stellar component with wavelength combined with the rising brightness of the dust, very small grains, and of the Active Galactic Nuclei (AGN) non thermal emission in the thermal infrared. The CIB is defined as the cosmic background at wavelengths longward of this minimum. An understanding of the nature and redshift distribution of the sources of the CIB, although relatively new, is an integral part of the understanding of the formation and evolution of galaxies.

The standard hierarchical model of structure formation has received strong observational support from observations of the large-scale distribution of galaxies, clusters, intergalactic clouds, combined with $\mathrm{CMB}$ anisotropies that constrain the initial large scale power spectrum within the concordance cosmological model framework. Scenarios for galaxy formation and evolution can be confronted with the very quickly rising set of observations of extragalactic sources at higher and higher redshifts. Nevertheless many critical questions remain open on the cooling of collapsed structures, angular momentum of galaxies, star formation efficiency, Initial Mass Function (IMF) of the stars formed, role of feedback mechanisms, the physics and role of merging and accretion in the construction of galaxies. 
As ultraluminous infrared galaxies were found to be often associated with mergers or interacting galaxies, it can be expected that the sources of the CIB carry critical information about the history of merging (e.g., Sanders \& Mirabel 1996; Genzel \& Cesarsky 2000). Because about half of the energy from extragalactic sources is in the CIB, the determination of the source of this energy (starbursts or massive black hole accretion in dust enshrouded AGNs) should shed some light on how galaxies evolve.

Since the discovery of the CIB in the COBE data (e.g., Hauser \& Dwek 2001), identifying the sources of the CIB, their redshift distribution, and nature progressed at increasing speed especially through multiwavelength analysis. This review attempts to give the broadband observational picture for the identification of the sources of the CIB. The detailed analysis of individual infrared galaxies is outside the scope of this review. We then discuss implications, both well-established ones and tentative ones, as well as directions for future work. The spectroscopy aspects are covered in the review by Solomon \& Vanden Bout (2005, in this volume). This is at present a fascinating but moving target! The coming decade will be a very rich one. New, very powerful long-wavelengths observation tools will bring many striking new results like the Spitzer observatory or the being built experiments like Herschel and ALMA. Throughout this review, the cosmology is fixed to $\Omega_{\Lambda}=0.7, \Omega_{\mathrm{m}}=0.3, \mathrm{H}_{0}=100 \mathrm{~h} \mathrm{~km} \mathrm{~s}^{-1} \mathrm{Mpc}^{-1}$ with $\mathrm{h}=0.65$.

\section{DUST IN THE LOCAL UNIVERSE}

A fraction of the stellar radiation produced in galaxies is absorbed by dust and re-radiated from mid-infrared to millimeter wavelengths. Understanding dust properties and the associated physics of the absorption and emission are thus essential. These determine the Spectral Energy Distribution (SED) of the galaxies.

\subsection{Dust Particles}

Small dust particles with sizes ranging from a nanometer to a fraction of micrometer are ubiquitous in the interstellar medium. They result from natural condensation in cool stellar atmospheres, supernovae, and the interstellar medium of the heavy elements produced by the nucleosynthesis in stars and released to the diffuse medium by late type stars and supernovae explosions. Interstellar grain models have been improved for 30 years in order to fit all observational constraints: elemental abundances of the heavy elements, UV, visible and infrared absorption and scattering properties, infrared emission, polarization properties of the absorbed and emitted light. The models include a mixture of amorphous silicate grains and carbonaceous grains, each with a wide size distribution ranging from molecules containing tens of atoms to large grains $\geq 0.1 \mu \mathrm{m}$ in diameter that can be coated with ices in dense clouds and/or organic residues (e.g., Désert et al. 1990; Li \& Draine 2001). It is now widely accepted that the smallest carbonaceous grains are Polycyclic Aromatic Hydrocarbons (PAHs) that emit a substantial fraction of the energy in a set of features between 3 and $17 \mu \mathrm{m}(3.3$, $6.2,7.7,8.6,11.3,12.7,16.3,17 \mu \mathrm{m}$ for the main ones) that used to be known as the UIB for Unidentified Infrared Bands. These features result from $\mathrm{C}-\mathrm{C}$ and $\mathrm{C}-\mathrm{H}$ stretching/bending vibrational bands excited by the absorption of a single UV or optical photon and are a good tracer of normal and moderately active star formation activity in spiral and irregular galaxies (e.g., Helou et al. 2000; Peeters et al. 2004). For radii $\mathrm{a} \geq 50 \AA$, the carbonaceous grains are often assumed to have graphitic properties. The so-called very small grains of the interstellar medium are small enough to have very low heat capacity, so their temperature are significantly affected by single-photon absorption. In the diffuse ISM of our Galaxy, they dominate the infrared emission for wavelengths smaller than about $80 \mu \mathrm{m}$. At longer wavelengths, the infrared spectrum is dominated by the emission of the larger grains at their equilibrium temperatures. Considering the energy density of the radiation in a galactic disc like ours, the temperature of the larger grains is rather low; 15 to $25 \mathrm{~K}$. For these grains, the far-infrared emissivity decreases roughly as the square of the wavelength. This in turn makes the temperature dependence on the radiation energy density $u$ very weak $\left(T \simeq u^{1 / 6}\right)$. For a galaxy like the Milky Way, the infrared part of the SED peaks at $170 \mu \mathrm{m}$ whereas for an Ultra Luminous Infrared Galaxy (ULIRG) it peaks at about 60 $\mu \mathrm{m}$ : a factor 3 in temperature for a factor $10^{3}$ 


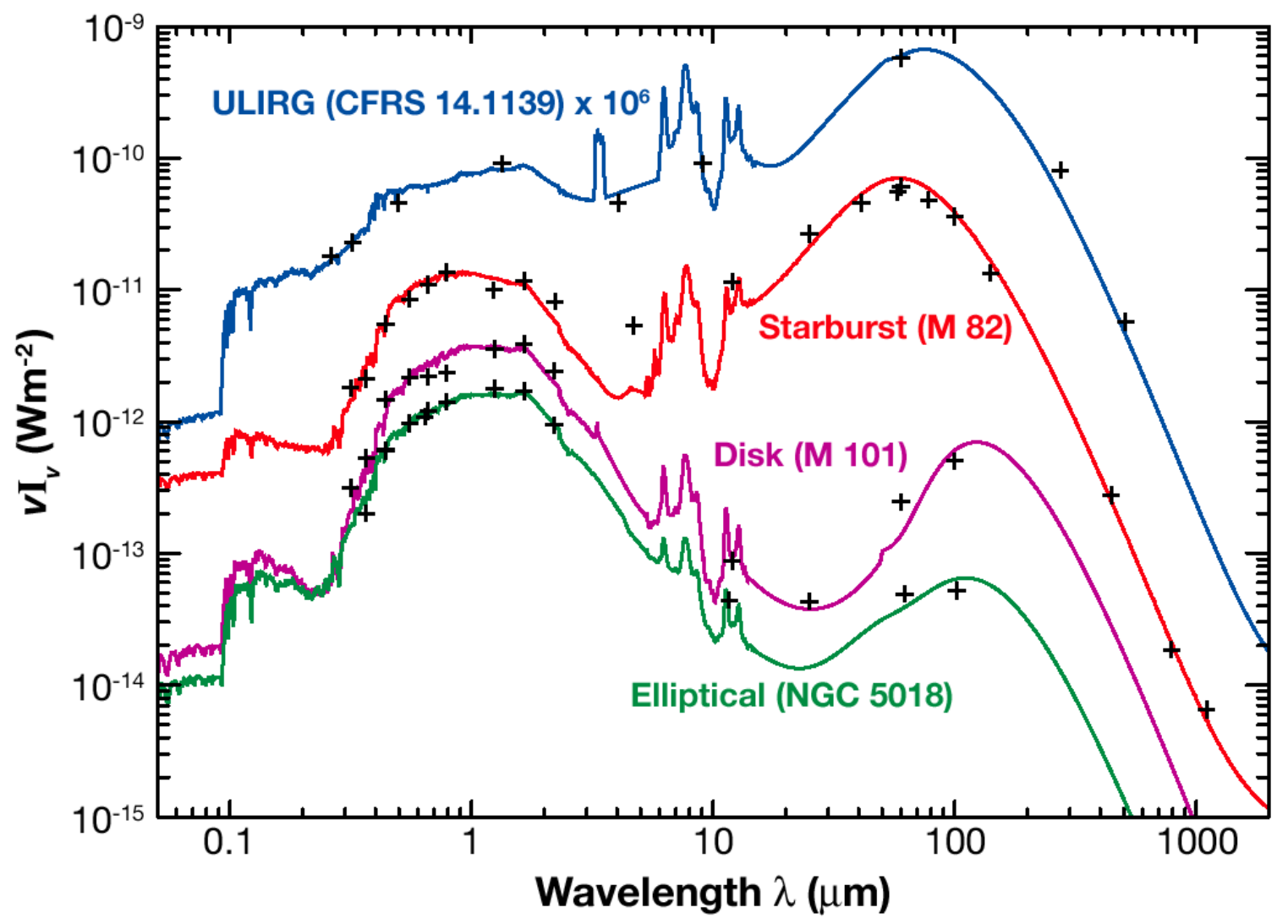

FIG. 1. - Spectral energy distributions of galaxies from UV to the millimeter. The ULIRG is observed at redshift $\mathrm{z}=0.66$ and is represented here in the rest-frame (from Galliano 2004).

in energy density or luminosity. At long wavelengths in the submillimeter and millimeter, the intensity should increase like $I_{\nu} \simeq \nu^{4}$.

\subsection{Extinction}

In our Galaxy, the extinction curve of the diffuse ISM has been known for a few decades. The average optical depth perpendicular to the disk of our Galaxy in the solar vicinity is small $\left(\mathrm{A}_{V}\right.$ $\simeq 0.2$ ) and typical of spiral galaxies. The average optical depth increases to a few in large molecular cloud complexes. It can become very large in galactic nuclei. Finally it should also be remembered that the optical depth in the UV is typically 10 times larger than that in optical wavelengths. The conversion of star light into infrared radiation will thus depend strongly on the location of the stars and their spectral types.

In external galaxies, modeling the extinction is very hard because it strongly depends on the geometric distribution of the ISM and of the chemical abundances. Simple models have been used to take this into account to first order. Galaxies can be modeled as an oblate ellipsoid where absorbers (dust) and sources (stars) are homogeneously mixed; the dust absorption can be computed in a "screen" or "sandwich" geometry (dust layers in front of the stars or sandwiched between two star layers). As a consequence, the reddening curve average over a whole galaxy appears to vary within a class of objects and between the different classes, from normal star-forming galaxies to highly concentrated starburst. It is thus very difficult to derive the total dust optical depth (e.g., Calzetti et al. 1994). In the local Universe, the average extinction per galaxy is quite low. About one third of the bolometric flux is emitted in the far-infrared, and this is typical of our Galaxy. 
In more actively star-forming galaxies, up to $70 \%$ of the bolometric flux is emitted in the farinfrared. In some of these, the starburst activity is mostly in the disk (like in M51). For a given total luminosity, the radiation energy density is lower than in the case of a starburst concentrated in a small volume in the nucleus. In this case, the dust will be hotter due to the larger energy density and the conversion of stellar light to infrared will be more efficient. Some ULIRGs emit more than $95 \%$ of their energy in the farinfrared (e.g., Arp 220). Such galaxies are very compact, dusty starbursts where dust optical depths are very large. In such galaxies, fine structure and recombination line ratios imply an equivalent "screen" dust extinction between $\mathrm{A}_{\mathrm{v}} \sim 5$ and 50. The result is that the SED is significantly distorted in the opposite way from the higher dust temperature (less mid-infrared emission). In the following, we will refer to "infrared galaxies" and to "optical galaxies" to designate galaxies in which the infrared emission, respectively optical emission dominates. Different typical spectra of galaxies are shown in Figure 1 from the UV to the millimeter. We clearly see the variation of the optical to infrared energy ratio as starburst activity increases.

\subsection{Local Infrared Galaxies}

A few very luminous infrared galaxies were observed in the seventies (Rieke \& Lebofsky 1979). Then IRAS satellite, launched in 1983 gave for the first time a proper census of the infrared emission of galaxies at low redshift. The Luminosity Function (LF) at 60 and $100 \mu \mathrm{m}$ is dominated by $L_{\star}$ spiral galaxies as could be expected - the reradiated stellar luminosity absorbed by dust. In addition, a high-luminosity tail of luminous galaxies was found (e.g., Sanders \& Mirabel 1996). This high-luminosity tail can be approximated by a power-law, $\Phi(L) \propto L_{\mathrm{IR}}^{2.35}$, which gives a space density for the most luminous infrared sources well in excess of predictions based on the optical LF. These sources comprise the Luminous Infrared Galaxies, LIRGs, and the ULIRGs with luminosities $11<\log \left(\mathrm{L}_{\mathrm{IR}} / \mathrm{L}_{\odot}\right)<12$ and $\log \left(\mathrm{L}_{\mathrm{IR}} / \mathrm{L}_{\odot}\right)>12$, respectively. These galaxies are often associated with interacting or merging, gas-rich disks. The fraction of strongly interacting/merger systems increases from $\sim 10 \%$ at $\log \left(\mathrm{L}_{\mathrm{IR}} / \mathrm{L}_{\odot}\right)=10.5-11$ to $\sim 100 \%$ at $\log \left(\mathrm{L}_{\mathrm{IR}} / \mathrm{L}_{\odot}\right)>12$. LIRGs are the site of intense starburst activity (about 10$100 \mathrm{M}_{\odot}$ year $^{-1}$ ) induced by the interaction and/or strong spiral structure. The ULIRG phase occurs near the end of the merging process when the two disks overlap. Such galaxies may be the precursors of Quasi Stellar Objects (QSOs; Sanders et al. 1988a, 1988b; Veilleux et al. 1995; Lutz et al. 1999). These objects have been the subject of intense debate concerning the nature of the dominant source of emission: starburst versus dustenshrouded AGN (e.g., Filipenko 1992; Sanders \& Mirabel 1996; Joseph 1999). Indeed, spectra show evidence of extremely large optical depth (heavily reddened continuum and large Balmer decrement) but also exhibit AGN-like high excitation fine-structure lines. We had to wait for ISO to clearly determine the power sources of ULIRGs. The difference between the mid-infrared spectra of starburst and AGNs is striking. Starburst are often characterized by strong, low-excitation, fine-structure lines, prominent PAH features and a weak $\lambda \geq 10 \mu \mathrm{m}$ continuum whereas AGNs display a highly excited emission line spectrum with weak or no $\mathrm{PAH}$ features, plus a strong mid-infrared continuum. It has been thus possible to build midinfrared diagnostic diagrams (e.g., Genzel et al. 1998; Laurent et al. 2000) that clearly separates starburst-dominated galaxies from AGNdominated galaxies. These diagrams demonstrate that ULIRGs appear to be composite objects, but star formation dominates in most objects. That is on average, $\geq 70 \%$ of the reradiated energy comes from starbursts and $\leq$ $30 \%$ comes from AGNs (Genzel et al. 1998; Lutz et al. 1998). However the fraction of AGN-powered objects increases with luminosity. About $15 \%$ of ULIRGs at luminosities below $2 \times 10^{12} \mathrm{~L}_{\odot}$ are mostly AGN powered, but this fraction increases to about half at higher luminosity.

All these well-studied LIRGs and ULIRGs are at low redshift. They do not dominate the energy production locally. As an example, the total infrared luminosity from these galaxies in the IRAS Bright Galaxy Sample accounts for only $\sim 6 \%$ of the infrared emission in the local Universe (Soifer \& Neugeubauer, 1991). As we will see, the situation changes dramatically at 
higher redshift where these galaxies fully dominate the infrared energy output.

\section{THE COSMIC INFRARED BACKGROUND}

The CIB is the infrared part of the extragalactic background, the radiation content of the Universe today produced by galaxies at all redshifts and seen as an isotropic extragalactic background radiation. Patridge \& Peebles (1967) predicted that observations of such a background could give powerful constraints on the cosmological evolution.

\subsection{General Observations and Direct Cosmological Implications}

The detection of the infrared part of the extragalactic background (the CIB for Cosmic Infrared Background) was the major objective of the DIRBE experiment aboard COBE. In fact, the CIB was first detected at long wavelengths by using the FIRAS spectrometer: $\lambda>200 \mu \mathrm{m}$ (Puget et al. 1996). The CIB has subsequently been detected by DIRBE at 2.4, 3.5, 100, 140, $240 \mu \mathrm{m}$ (see Hauser \& Dwek 2001 and Kashlinsky 2005 for two reviews). The extragalactic background at 2.4 and $3.5 \mu \mathrm{m}$ is significantly larger than that predicted by the integrated galaxy counts and their extrapolation. Similarly, the extragalactic background in the optical has been finally evaluated in combining several methods by Bernstein et al. (2002) and found to be larger than the value given by the integrated fluxes of galaxies by a factor larger than 2 . In the mid-infrared, the interplanetary zodiacal dust emission is so strong that only upper limits were obtained by DIRBE. The combination of number counts by ISO/ISOCAM at $15 \mu \mathrm{m}$ (see Elbaz \& Cesarsky 2003) and by Spitzer at $24 \mu \mathrm{m}$ (e.g., Papovich et al. 2004) giving lower limits, with the observations of $\mathrm{TeV}$ gamma ray emission from distant AGNs (e.g., Renault et al. 2001; Dwek \& Krennrich 2005), gives a good measurement of the background at these wavelengths. The full cosmic background spectrum is shown in Figure 2 Only most recent and strongly constraining measurements have been plotted for clarity.

Figure 2 clearly shows that the optical and infrared cosmic backgrounds are well separated. The first surprising result is that the power in the infrared is comparable to the power in the optical. In contrast, we know that locally, the infrared output of galaxies is only one third of the optical output. This implies that infrared galaxies grow more luminous with increasing $z$ faster than do optical galaxies. A second important property to note is that the slope of the long wavelength part of the CIB, $I_{\nu} \propto \nu^{1.4}$ (Gispert et al. 2000), is much less steep than the long wavelengths spectrum of galaxies (as illustrated in Figure 2 with the M82 SED). This implies that the millimeter CIB is not due to the millimeter emission of the galaxies that account for the peak of the CIB $(\simeq 150 \mu \mathrm{m})$. The implications in terms of energy output have been drawn by, e.g. Gispert et al. (2000). The infrared production rate per comoving unit volume $(a)$ evolves faster between redshift zero and 1 than the optical one and $(b)$ has to stay roughly constant at higher redshifts up to redshift 3 at least.

\subsection{The Status of Deep Surveys: Resolved Fraction of the $>10 \mu \mathrm{m} \mathrm{CIB}$}

Many surveys from the mid-infrared to the millimeter have aimed to resolve the CIB into discrete sources. From short to long wavelengths the significant surveys are the following:

- ISOCAM $15 \mu \mathrm{m}$ : Three kinds of surveys have been done. The shallowest is the ELAIS survey (European Large-Area ISO Survey, Oliver et al. 2000). The deepest is the survey in the HDF-N (Aussel et al. 1999) as well as the surveys in the direction of the galaxy clusters (e.g. Metcalfe et al. 2003). Altogether, about 1000 galaxies were detected. Above a sensitivity limit of $50 \mu \mathrm{Jy}$, they produce a $15 \mu \mathrm{m}$ extragalactic background light of $(2.4 \pm$ 0.5) $\mathrm{nW} \mathrm{m}^{-2} \mathrm{sr}^{-1}$ (Elbaz et al. 2002). This accounts for about $80 \%$ of the CIB at $15 \mu \mathrm{m}$ based on the simplest extrapolation of the counts.

- Spitzer $24 \mu \mathrm{m}$ : Spitzer surveys are ongoing, but more than $10^{5}$ sources have already been detected at $24 \mu \mathrm{m}$. Integrating the first number counts down to 60 $\mu \mathrm{Jy}$, a lower limit to the CIB at $24 \mu \mathrm{m}$ of $(1.9 \pm 0.6) \mathrm{nW} \mathrm{m}^{-2} \mathrm{sr}^{-1}$ is derived $(\mathrm{Pa}-$ povich et al. 2004). This accounts for 


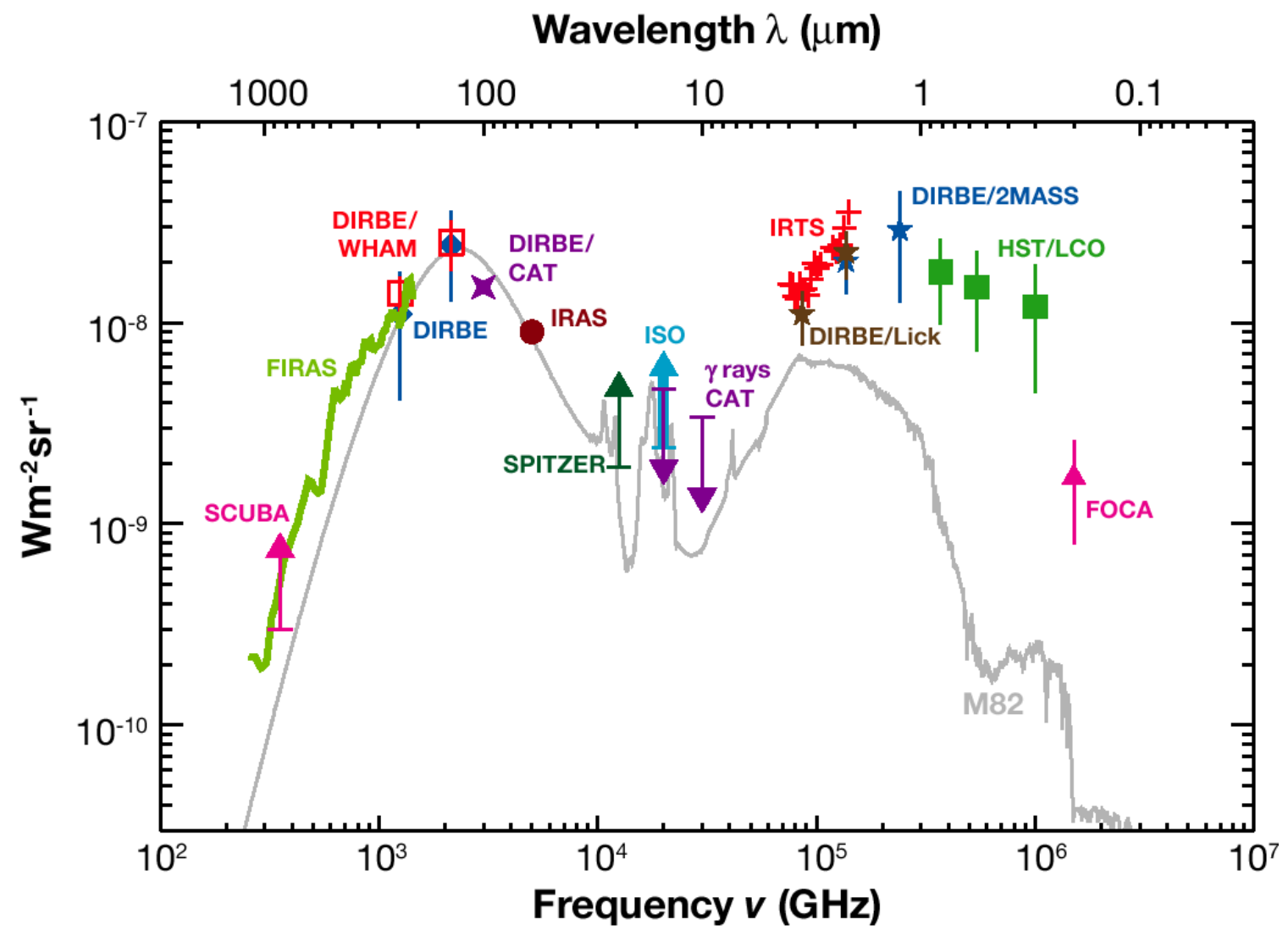

FIG. 2.- The extragalactic background over three decades in frequency from the near UV to millimeter wavelengths. Only strongly constraining measurements have been reported. We show for comparison in grey an SED of M82 (Chanial, 2003) - a starburst galaxy at $\mathrm{L}=3 \times 10^{10} \mathrm{~L} \odot-$ normalized to the peak of the CIB at $140 \mu \mathrm{m}$. References for data points are given in Table

TABLE 1

Extragalactic BaCKGround REFEREnCES For Figure[2]

\begin{tabular}{|c|c|c|c|}
\hline Wavelength $(\mu \mathbf{m})$ & Experiment & Measurement & Reference \\
\hline$\overline{0.2}$ & FOCA & Number counts \& model & Armand et al. 1994 \\
\hline $0.30,0.56,0.81$ & $\begin{array}{c}\text { HST/Las } \\
\text { Campanas Obs. }\end{array}$ & Diffuse emission & $\begin{array}{l}\text { Bernstein et al. } 2002 \\
\text { Mattila } 2003\end{array}$ \\
\hline $2.2<\lambda<4$ & IRTS & Diffuse emission & Matsumoto et al. 2005 \\
\hline $2.2,3.3$ & DIRBE/Lick & Diffuse emission & Gorjian et al. 2000 \\
\hline $1.25,2.2$ & DIRBE/2MASS & Diffuse emission & $\begin{array}{l}\text { Wright 2001 } \\
\text { Cambrésy et al. } 2001\end{array}$ \\
\hline 10,15 & CAT & $\gamma$-rays & Renault et al. 2001 \\
\hline 15 & ISO/ISOCAM & Number counts & Elbaz et al. 1999 \\
\hline 24 & Spitzer/MIPS & Number counts & Papovich et al. 2004 \\
\hline 60 & IRAS & Power spectrum & $\begin{array}{l}\text { Miville-Deschênes et } \\
\text { al. } 2001\end{array}$ \\
\hline 100 & DIRBE & Diffuse emission & Renault et al. 2001 \\
\hline 140,240 & DIRBE/WHAM & Diffuse emission & Lagache et al. 2000 \\
\hline 140,240 & DIRBE & Diffuse emission & Hauser et al. 1998 \\
\hline 850 & SCUBA & Number counts & Smail et al. 2002 \\
\hline $200<\lambda<1200$ & FIRAS & Diffuse emission & Lagache et al. 2000 \\
\hline
\end{tabular}




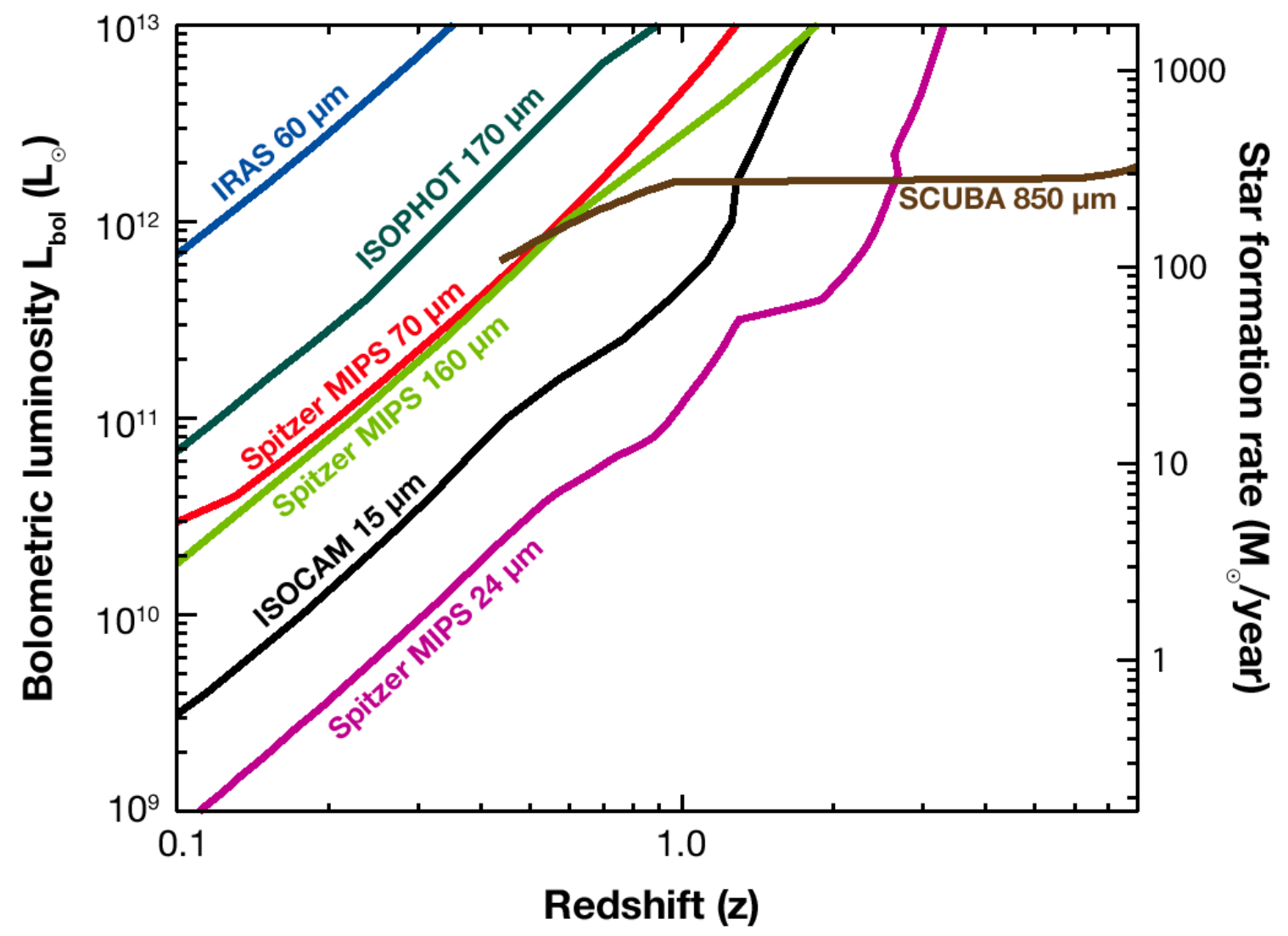

FIG. 3.- Sensitivity to the bolometric luminosity and star-formation rate, assuming star forming galaxies of various infrared and submillimeter experiments. Detections of at least 10 sources in the surveys can be expected in the areas above the curves. We assumed the scenario of a typical deep survey (when available). IRAS $60 \mu \mathrm{m}\left(S_{\nu}>1 \mathrm{Jy}\right.$, all sky); ISOCAM $15 \mu \mathrm{m}\left(S_{\nu}>250 \mu \mathrm{Jy}, 2 \mathrm{Sq}\right.$. Deg.); ISOPHOT $170 \mu \mathrm{m}\left(S_{\nu}>180 \mathrm{mJy}, 5\right.$ Sq. Deg. $)$ Spitzer/MIPS $24 \mu \mathrm{m}$ $\left(S_{\nu}>80 \mu\right.$ Jy, 5 Sq. Deg.); Spitzer/MIPS $70 \mu \mathrm{m}\left(S_{\nu}>25 \mathrm{mJy}, 5\right.$ Sq. Deg. $)$; Spitzer/MIPS $160 \mu \mathrm{m}\left(S_{\nu}>50 \mathrm{mJy}, 5\right.$ Sq. Deg.); $S C U B A 850 \mu \mathrm{m}\left(S_{\nu}>1 \mathrm{mJy}, 1 \mathrm{Sq}\right.$. Deg.). This plot makes use of the Lagache et al. (2004) model (see the Appendix).

- Spitzer $70 \mu \mathrm{m}$ : Counts have been derived down to $15 \mathrm{mJy}$. Integrating these counts corresponds $0.95 \mathrm{nW} \mathrm{m}^{-2} \mathrm{sr}^{-1}$ which explains $\sim 20 \%$ of the CIB at $70 \mu \mathrm{m}$ as derived from the Lagache et al. (2004) model (Dole et al. 2004a).

- ISOPHOT $90 \mu \mathrm{m}$ : The most relevant data comes from the ELAIS survey that covers about 12 square degrees at $90 \mu \mathrm{m}$. Counts have been obtained down to 95 mJy (Héraudeau et al. 2004) resolving less than $5 \%$ of the CIB as derived from DIRBE by Renault et al. (2001).

- Spitzer $160 \mu \mathrm{m}$ : First counts are derived down to $50 \mathrm{mJy}$. The integral of these counts corresponds to $1.4 \mathrm{nW} \mathrm{m}{ }^{-2} \mathrm{sr}^{-1}$, which explain about $7 \%$ of the CIB at 160 $\mu \mathrm{m}$ (Dole et al. 2004a).

- ISOPHOT $170 \mu \mathrm{m}$ : Two main surveys have been conducted: the FIRBACK (Lagache \& Dole 2001) and the Lockman hole (Kawara et al. 2004) surveys that covering about 5 square degrees. Counts down to 135 mJy contribute to less than $5 \%$ of the CIB (Dole et al. 2001).

- SCUBA $450 \mu \mathrm{m}$ : Deep surveys at $450 \mu \mathrm{m}$ are very hard to conduct from the ground. A few galaxies are detected between 10 and $50 \mathrm{mJy}$ in deep surveys. Number counts down to $10 \mathrm{mJy}$ give a lower limit on the CIB of about $(0.7 \pm 0.4) \mathrm{nW} \mathrm{m}^{-2}$ $\mathrm{sr}^{-1}$ (Smail et al. 2002). This resolves 
about $15 \%$ of the CIB at $450 \mu \mathrm{m}$.

- SCUBA $850 \mu \mathrm{m}$ : Over $500 \operatorname{arcmin}^{2}$ of blank sky has been surveyed by several groups using SCUBA. The observations range from an extremely deep survey in the area of the HDF-N to a wider-field shallower survey. Also about $40 \operatorname{arcmin}^{2}$ of lensed cluster fields have been observed in which 17 sources have been detected. Fluxes range from about 0.5 to $8 \mathrm{mJy}$. The flux density in the resolved submillimeter population down to $1 \mathrm{mJy}$ is $(0.3$ $\pm 0.1) \mathrm{nW} \mathrm{m}^{-2} \mathrm{sr}^{-1}$ (Smail et al. 2002). This account for $60 \%$ of the CIB at 850 $\mu \mathrm{m}$. Note that in deep surveys, sources with $\mathrm{S}_{850} \geq 3$ mJy contribute to $\sim 30 \%$ of the CIB.

- MAMBO 1200 m: Deep blank sky areas surveyed by MAMBO cover about 500 $\operatorname{arcmin}^{2}$. Number counts are given by Greve et al. (2004). By integrating the counts from 2.25 to $5.75 \mathrm{mJy}$, the resolved CIB is about $0.02 \mathrm{nW} \mathrm{m}^{-2} \mathrm{sr}^{-1}$, or about $10 \%$ of the total CIB at $1200 \mu \mathrm{m}$.

Figure 3 shows the capabilities of the different surveys to find distant LIRGs. Spitzer observations at $24 \mu \mathrm{m}$ are the most powerful tool to find LIRGs up to $z \sim 2.2$; ISOCAM was limited at $z \sim 1.2$. Distant ULIRGs are found by deep and large surveys at 24 and $850 \mu \mathrm{m}$. Note that capabilities have been computed using the model of Lagache et al. (2004). This empirical model is based on only two populations of galaxies; it aims only to model the redshift evolution of the average population. It reproduces all the observations from mid-infrared to the millimeter (see Appendix). Lewis et al. (2005) showed that a more sophisticated, bivariate SED does not much change the average properties although it does significantly change the dispersion. The Lagache et al. (2004) model is thus used in this paper as a tool to discuss observations and predictions.

\subsection{Redshift Contribution to the CIB}

From Figure 2] we see that contributions from galaxies at various redshifts are needed to fill the CIB SED shape. The bulk of the CIB in energy, i.e., the peak at about $150 \mu \mathrm{m}$, is not resolved in individual sources but one
TABLE 2

Redshift AT Which THE COSMIC INFRARED BACKGROUND IS RESOLVED AT 20,50 OR 80\% (FROM THE MODEL OF LAGACHE ET AL. 2004).

\begin{tabular}{llll}
\hline \hline Wavelength & $20 \%$ & $50 \%$ & $80 \%$ \\
\hline $15 \mu \mathrm{m}$ & & & \\
$24 \mu \mathrm{m}$ & 0.5 & 1.0 & 1.3 \\
$70 \mu \mathrm{m}$ & 0.5 & 1.3 & 2.0 \\
$100 \mu \mathrm{m}$ & 0.5 & 1.0 & 1.5 \\
$160 \mu \mathrm{m}$ & 0.7 & 1.0 & 1.7 \\
$350 \mu \mathrm{m}$ & 0.7 & 1.3 & 2.0 \\
$850 \mu \mathrm{m}$ & 1.0 & 2.0 & 3.0 \\
$1.4 \mathrm{~mm}$ & 2.0 & 3.0 & 4.0 \\
$2.1 \mathrm{~mm}$ & 2.5 & 3.5 & 4.5 \\
& 2.0 & 3.5 & 5.0 \\
\hline
\end{tabular}

dominant contribution at the CIB peak can be inferred from the ISOCAM deep surveys. ISOCAM galaxies with a median redshift of $\sim 0.7$ resolve about $80 \%$ of the CIB at $15 \mu \mathrm{m}$. Elbaz et al. (2002) separate the $15 \mu \mathrm{m}$ galaxies into different classes (ULIRGs, LIRGs, Starbursts, normal galaxies and AGNs) and extrapolate the $15 \mu \mathrm{m}$ fluxes to $140 \mu \mathrm{m}$ using

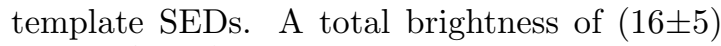
$\mathrm{nW} \mathrm{m} \mathrm{m}^{-2} \mathrm{sr}^{-1}$ is found, which makes up about two thirds of the CIB observed at $140 \mu \mathrm{m}$ by COBE/DIRBE. Hence, the galaxies detected by ISOCAM are responsible for a large fraction of energy of the CIB. About one half of the $140 \mu \mathrm{m} \mathrm{CIB}$ is due to LIRGs and about one third to ULIRGs. However, these ISOCAM galaxies make little contribution to the CIB in the millimeter and submillimeter. There, the CIB must be dominated by galaxies at rather high redshift for which the SED peak has been shifted. The redshift contribution to the CIB is illustrated in Figure 4 We clearly see that the submillimeter/millimeter CIB contains information on the total energy output by the high-redshift galaxies $\left(\begin{array}{l}z>2\end{array}\right)$. This is supported by the redshift distribution of the SCUBA sources at $850 \mu \mathrm{m}$ with $\mathrm{S}_{850} \geq 3$ mJy that make about $30 \%$ of the CIB and have a median redshift of 2.2 (Chapman et al. 2005).

Figure 5 shows the fraction of resolved CIB as a function of redshift for selected wavelengths. Fifty percents of the CIB is due to galaxies at redshift below 1 at 15 and $70 \mu \mathrm{m}, 1.3$ at 24 and $160 \mu \mathrm{m}, 2$ at $350 \mu \mathrm{m}, 3$ at $850 \mu \mathrm{m}$ and 


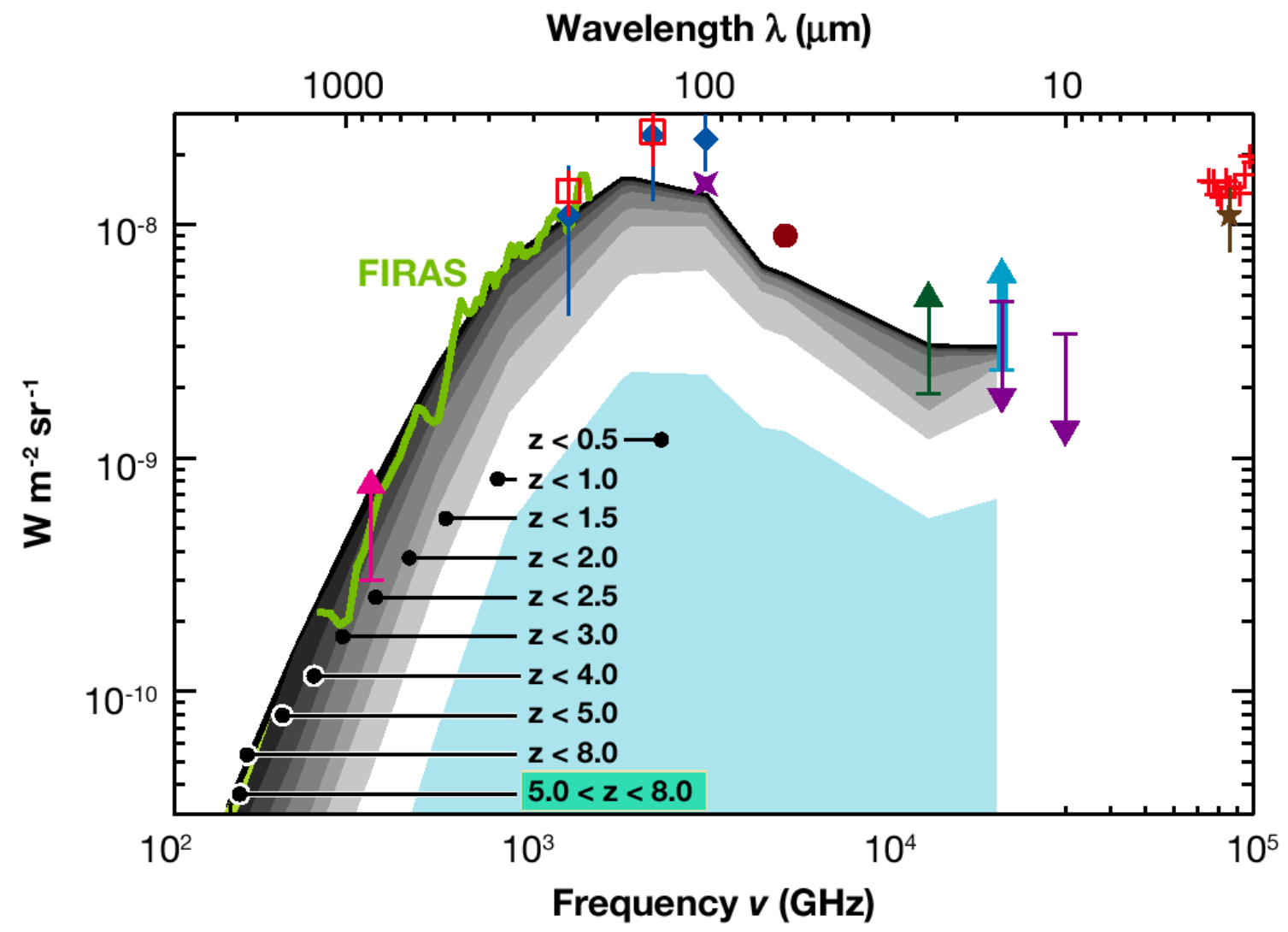

Fig. 4.- Cumulative contribution to the CIB of galaxies at various redshifts from 0.5 to 8 , from the model of Lagache et al. (2004). Measurements of the CIB are reported with the same symbols as in Figure 2

3.5 at $2000 \mu \mathrm{m}$ (see also Table 2). It is clear that from the far-infrared to the millimeter, the CIB at longer wavelengths probes sources at higher redshifts.

From Section 3.2 we see that the most constraining surveys in term of resolving the CIB are those at 15,24 and $850 \mu \mathrm{m}$. Moreover, the capabilities of these surveys to find high- $z$ objects are the best among all other existing surveys (see Figure 31). These surveys probe the CIB in well-defined and distinct redshift ranges, with median redshifts of 0.7 (Liang et al. 2004), $\sim 1$ (Caputi et al. 2005 and L. Yan, private communication), and 2.2 (Chapman et al. 2005) at 15,24 and $850 \mu \mathrm{m}$, respectively. Such welldefined redshift ranges can be understood by looking at the K-correction. The K-correction is defined as:

$$
K(L, z)=\frac{L_{\nu}(1+z)}{L_{\nu}(z=0)}
$$

where $L_{\nu}(z=0)$ is the rest-frame luminosity. This correction is specific to the spectrum of the population considered at a given luminosity and redshift. Figure [ 6 shows the K-correction at $15,24,70,160$ and $850 \mu \mathrm{m}$. The broad plateau observed around $z=1$ at $15 \mu \mathrm{m}$ and around $z=2$ at $24 \mu \mathrm{m}$ is caused by the PAHs' features. At longer wavelengths, the slow decrease of the $\mathrm{K}$-correction is caused by the shape of the starburst spectra around the peak of their emission. At $850 \mu \mathrm{m}$, the monotonic rise favors the detection of high- $z$ objects.

\section{GALAXIES AT REDSHIFTS $0.5 \leq Z \leq 1.5$}

At the time this review was written, most of the detailed informations on dusty galaxies in the $0.5 \leq z \leq 1.5$ redshift range comes from 


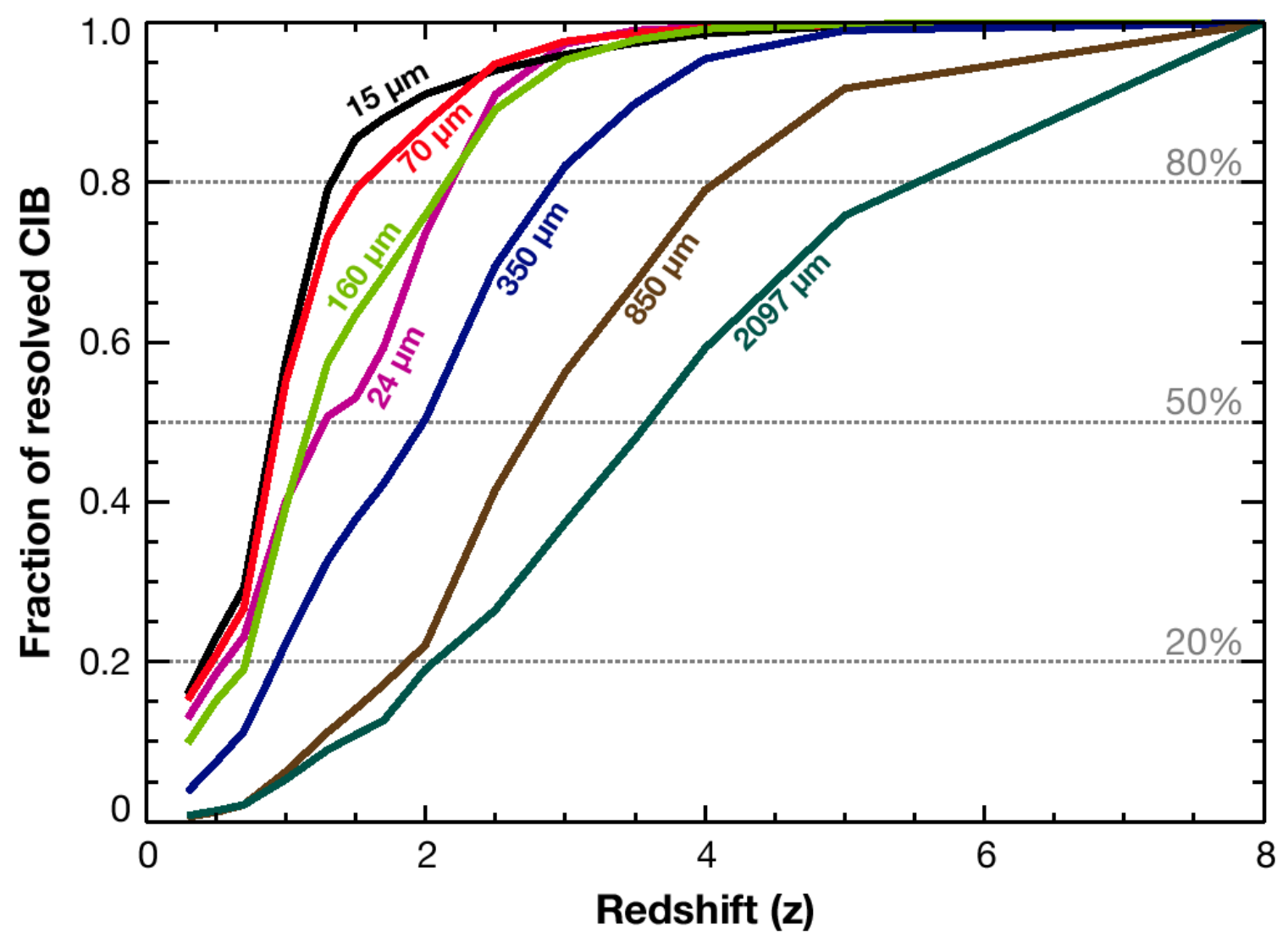

FIG. 5.- Cumulative fraction of the CIB content as a function of redshift for various wavelengths, from the model of Lagache et al. (2004).

galaxies selected with the ISOCAM cosmological surveys and the multi-wavelength analysis of detected sources. The ISOCAM extragalactic surveys were performed with two filters, LW2 $(5-8.5 \mu \mathrm{m})$ and LW3 $(12-18 \mu \mathrm{m})$ centered at 6.75 and $15 \mu \mathrm{m}$, respectively. However, because of star contamination and because the stellar light dominates the flux from galaxies in the $6.75 \mu \mathrm{m}$ band above redshift 0.4 , only the $15 \mu \mathrm{m}$ surveys are relevant here. Shallow, deep and ultra-deep surveys were performed in various fields including the Lockman hole, Marano, northern and southern Hubble Deep Field (HDF), and Canada-France Redshift Survey (CFRS) (e.g., Aussel et al. 1999; Flores et al. 1999; Lari et al. 2001; Gruppioni et al. 2002; Mann et al. 2002; Elbaz \& Cesarsky 2003; Sato et al. 2003). Deeper images have been made in the direction of distant clusters (e.g., Metcalfe et al. 2003). Finally, the bright end of the lu- minosity function was explored by the ELAIS survey (e.g., Oliver et al. 2000). The deepest surveys reach a completeness limit of about $100 \mu \mathrm{Jy}$ at $15 \mu \mathrm{m}$ (without lensing). The most relevant data to this section are the deep and ultra-deep surveys.

\subsection{Detailed Properties}

To find out the nature and redshift distribution of the $15 \mu \mathrm{m}$ deep survey sources, many followup observations have been conducted including HST imaging and VLT spectroscopy. With a point-spread function full width at half of maximum of $4.6 \operatorname{arcsec}$ at $15 \mu \mathrm{m}$, optical counterparts are easily identified. Redshifts are found using emission and/or absorption lines. From field to field, the median redshift varies from 0.52 to 0.8 , a quite large variation due to sample variance. Each field clearly exhibits one or two redshift peaks, with velocity disper- 


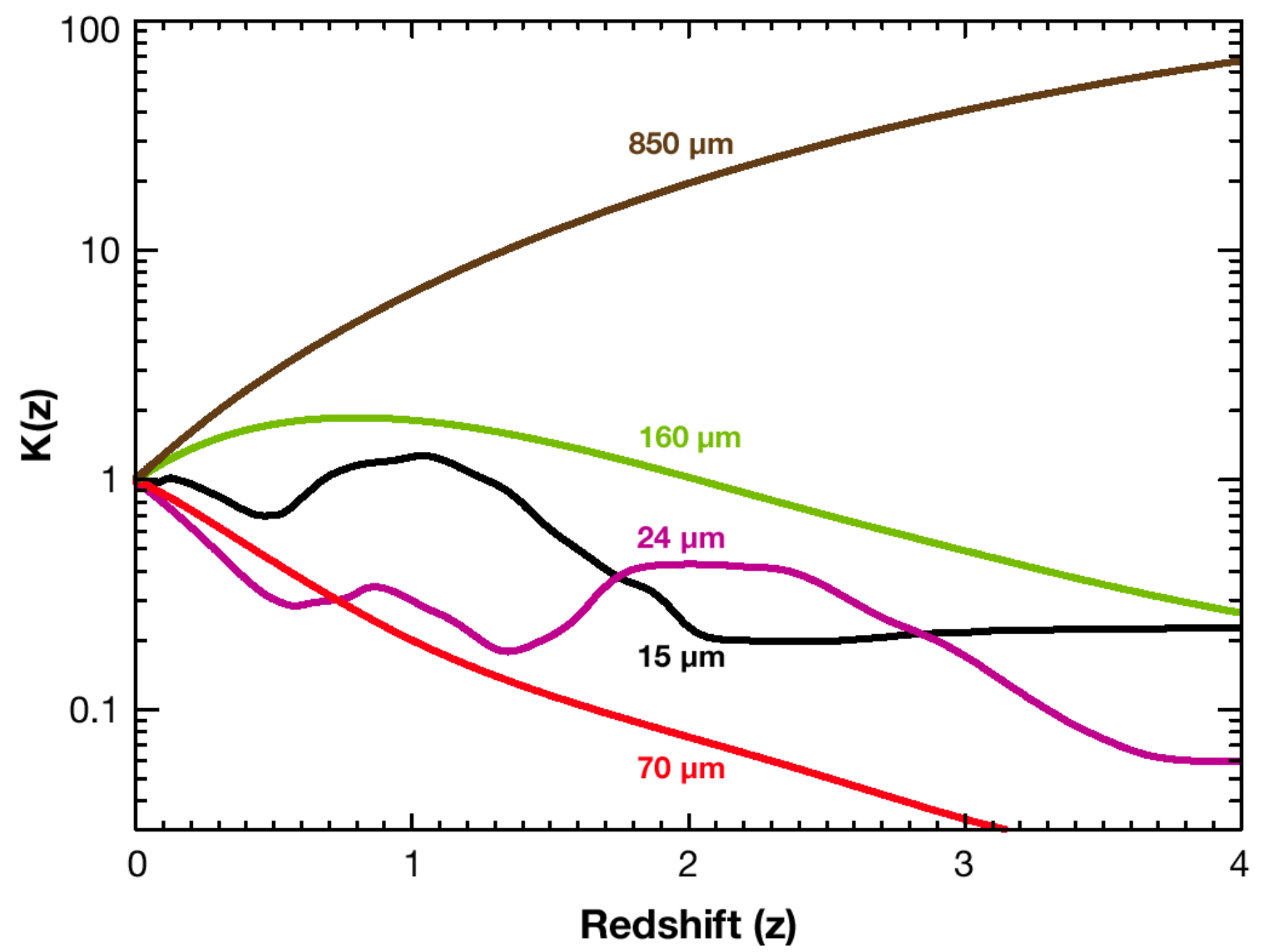

FIG. 6.- K-correction at 15, 24, 70 and 160 and $850 \mu \mathrm{m}$ for a typical LIRG with $\mathrm{L}=2 \times 10^{11} \mathrm{~L} \odot$ (from the model of Lagache et al. 2004).

sion characteristic of clusters or galaxy groups. Most of ISOCAM galaxies have redshifts between $\sim 0.3$ and 1.2, consistent with Figure 3 About $85 \%$ of the ISO galaxies show obvious strong emission lines (e.g., [OII] 3723, $\mathrm{H}_{\gamma}, \mathrm{H}_{\beta}$, [OIII] 4959, 5007). These lines can be used as a diagnostic of the source of ionization and to distinguish the HII-region like objects from the Seyferts and LINERs. Most of the objects are found to be consistent with HII regions, e.g., from Liang et al. (2004) and exhibit low ionization level $\left([\mathrm{OIII}] / \mathrm{H}_{\beta}<3\right)$. From emission lines studies, the AGN fraction is quite low, $\sim 20 \%$. This is consistent with X-ray observations of ISOCAM sources showing that AGNs contribute at most $17 \pm 6 \%$ of the total midinfrared flux (Fadda et al. 2002). Assuming template SEDs typical of star-forming and starburst galaxies, $15 \mu \mathrm{m}$ fluxes can be converted into total infrared luminosities, $\mathrm{L}_{\mathrm{IR}}$ (between 8 and $1000 \mu \mathrm{m}$ ). About $75 \%$ of the galaxies dominated by the star formation are either LIRGs or ULIRGs. The remaining $25 \%$ are nearly equally distributed among either "starbursts" $\left(10^{10}<\mathrm{L}_{\mathrm{IR}}<10^{11} \mathrm{~L}_{\odot}\right)$ or "normal" $\left(\mathrm{L}_{\mathrm{IR}}<\right.$ $\left.10^{10} \mathrm{~L}_{\odot}\right)$ galaxies. The median luminosity is about $3 \times 10^{11} \mathrm{~L}_{\odot}$. ULIRGs and LIRGs contribute to about $17 \%$ and $44 \%$ to the CIB at 15 $\mu \mathrm{m}$, respectively (Elbaz et al. 2002). This suggests that the star formation density at $z<1$ is dominated by the abundant population of LIRGs. As will be shown later, this has important consequences for the evolution of galaxies. Because of large extinction in LIRGs and ULIRGs, the infrared data provide more robust SFR estimate than UV tracers. The extinction factor in LIRGs averages to $\mathrm{A}_{V} \sim 2.8$ at $z \sim 0.7$ (Flores et al. 2004). It is much higher than 
that of the local star-forming galaxies for which the median is 0.86 (Kennicutt 1992). Assuming continuous burst of age 10-100 Myr, solar abundance, and a Salpeter initial mass function, the SFR can be derived from the infrared luminosities (Kennicutt 1998):

$$
\operatorname{SFR}\left(M_{\odot} / y r\right)=1.71 \times 10^{-10} L_{I R}[8-1000 \mu \mathrm{m}]\left(\mathrm{L}_{\odot}\right)
$$

Thus typical LIRGs form stars at $\geq 20 \mathrm{M}_{\odot}$ year $^{-1}$. The median SFR for the $15 \mu \mathrm{m}$ galaxies is about $50 \mathrm{M}_{\odot}$ year $^{-1}$, a substantial factor larger than that found for faint-optically selected galaxies in the same redshift range.

The other fundamental parameter characterizing the sources of the peak of the infrared background is their stellar mass content that traces the integral of the past star formation activity in the galaxies and is a natural complement to the instantaneous rate of star formation. The stellar masses can be obtained using spectral synthesis code modeling of the UV-optical-near infrared data or, more simply using the mass-to-luminosity ratio in the K-band. The derived stellar masses for the bulk of ISOCAM galaxies range from about $10^{10}$ to $3 \times 10^{11} \mathrm{M}_{\odot}$, compared to $1.8 \times 10^{11}$ $\mathrm{M}_{\odot}$ for the Milky Way. As expected from the selection based on the LW3 flux limit and thus on the SFR - masses do not show significant correlation with redshift (Franceschini et al. 2003). An estimate of the time spent in the starburst state can be obtained by comparing the rate of ongoing star formation (SFR) with the total mass of already formed stars: $t$ years $]=M / \mathrm{SFR}$. Assuming a constant SFR, $t$ is the timescale to double the stellar mass. For LIRGs at $z>0.4, t$ ranges from 0.1 to 1.1 Gy with a median of about 0.8 Gyr (Franceschini et al. 2003; Hammer et al. 2005). From $z=1$ to $z=0.4$ (i.e., $3.3 \mathrm{Gyr}$ ), this newly formed stellar mass in LIRGs corresponds to about $60 \%$ of the $z=1$ total mass of intermediate mass galaxies. The LIRGs are shown to actively build up their metal content. In a detailed study, Liang et al. (2004) show that, on average, the metal abundance of LIRGs is less than half of the $z \sim 0$ disks with comparable brightness. Expressed differently, at a given metal abundance, all distant LIRGs show much larger B luminosities than local disks. Assuming that LIRGs eventually evolve into the local massive disk galaxies, Liang et al. (2004) suggest that LIRGs form nearly half of their metals and stars since $z \sim 1$.

Finally, morphological classification of distant LIRGs is essential to understand their formation and evolution. Zheng et al. (2004) performed a detailed analysis of morphology, photometry, and color distribution of $36(0.4<$ $z<1.2$ ) ISOCAM galaxies using HST images. Thirty-six percents of LIRGs are classified as disk galaxies with Hubble type from Sab to Sd; $25 \%$ show concentrated light distributions and are classified as Luminous Compact Galaxies (LCGs); 22\% display complex morphology and clumpy light distributions and are classified as irregular galaxies; only $17 \%$ are major ongoing mergers showing multiple components and apparent tidal tails. This is clearly different from the local optical sample of Nakamura et al. (2004) in the same mass range in which $27 \%, 70 \%,<2 \%, 3 \%$ and $<2 \%$ are $\mathrm{E} / \mathrm{S} 0$, spirals, LCGs, irregulars and major mergers respectively. Consequences for galaxy evolution will be given in Section 4.3 For most compact LIRGs, the color maps reveal a central region strikingly bluer than the outer regions. These blue central regions have a similar size to that of bulges and a color comparable to that of starforming regions. Because the bulge/central region in local spiral is relatively red, such a blue core structure could imply that the galaxy was forming the bulge (consistent with Hammer et al. 2001). It should be noticed that they find all LIRGs distributed along a sequence that relates their central color to their compactness. This is expected if star formation occurs first in the center (bulge) and gradually migrate to the outskirts (disk), leading to redder colors of the central regions as the disk stars were forming.

\subsection{Cosmic Evolution}

Another remarkable property of the $15 \mu \mathrm{m}$ sources is their extremely high rates of evolution with redshift exceeding those measured for galaxies at other wavelengths and comparable to or larger than the evolution rates observed for quasars. Number counts at $15 \mu \mathrm{m}$ show a prominent bump peaking at about $0.4 \mathrm{mJy}$. At the peak of the bump, the counts are one order of magnitude above the non-evolution 
models. In fact, data require a combination of a $(1+z)^{3}$ luminosity evolution and $(1+z)^{3}$ density evolution for the starburst component at redshift lower than 0.9 to fit the strong evolution. Although it has not been possible with ISOCAM to probe in detail the evolution of the infrared luminosity function, Spitzer data at $24 \mu \mathrm{m}$ give for the first time tight constraints up to redshift 1.2 (Le Floc'h et al. 2005; Pérez-González et al. 2005). A strong evolution is noticeable and requires a shift of the characteristic luminosity $L^{\star}$ by a factor $(1+z)^{4.0 \pm 0.5}$. Le Floc'h et al. (2005) find that the LIRGs and ULIRGs become the dominant population contributing to the comoving infrared energy density beyond $z \sim 0.5-0.6$ and represent $70 \%$ of the star-forming activity at $z \sim 1$. The comoving luminosity density produced by luminous infrared galaxies was more than 10 times larger at $z \sim 1$ than in the local Universe. For comparison, the B-band luminosity density was only three times larger at $z=1$ than today. Such a large number density of LIRGs in the distant Universe could be caused by episodic and violent starformation events, superimposed on relatively small levels of star formation activity. This idea has emerged in 1977 (Toomre 1977) and is fully developed in Hammer et al. (2005). These events can be associated to major changes in the galaxy morphologies. The rapid decline of the luminosity density from $z=1$ is only partially due to the decreasing frequency of major merger events. Bell et al. (2005) showed that the SFR density at $z \sim 0.7$ is dominated by morphologically normal spiral, E/S0 and irregular galaxies $(\geq 70 \%)$, while clearly interacting galaxies account for $<30 \%$. The dominent driver of the decline is a strong decrease in SFR in morphologically undisturbed galaxies. This could be due, for example, to gas consumption or to the decrease of weak interactions with small satellites that could trigger the star formation through bars and spiral arms.

Locally $0.5 \%$ of galaxies with $\mathrm{L}_{\mathrm{V}}>10^{10} \mathrm{~L}_{\odot}$ have SEDs typical of LIRGs. This changes dramatically at higher redshift: in deep surveys, ISO detect about $15 \%$ of the $\mathrm{M}_{B} \leq-20$ galaxies (LIRGs, Hammer et al. 2005) and Spitzer de- tect about $30 \%$ of field galaxies (Starbursts and LIRGs, Bell et al. 2005). Thus the two populations (optical and LIR galaxies) overlap more at high $z$.

\subsection{Towards a Scenario of Recent Bulge and Disk Formation in Intermediate-Mass Spirals}

Because a significant part of recent star formation takes place in LIRGs, any overall picture of galaxy evolution requires a detailed panchromatic study. Optical/spectral properties of LIRGs are similar to those of other galaxies and only infrared measurements are able to describe how the star formation is distributed between the different galaxy types. Thus a complete study has to link the star formation revealed in the infrared to the morphological changes seen in the optical. This has been done by Hammer et al. (2005) using HST, ISO, VLA and VLT observations of the CFRS. A detailed comparison of the morphologies of distant $(0.4<\mathrm{z}<1.2)$ galaxies with the local galaxies shows the complete vanishing of the LCGs in the local Universe (by a factor $\sim 10$ ) and the decrease of mergers and irregulars (by a factor $\sim 4$ ). Almost all distant galaxies have much bluer central colors than local bulges, probably as a result of active star formation in the $1 \mathrm{kpc}$ central region of most distant spirals. This supports a relatively recent formation of bulges in many present-day spirals. This simultaneous changes in galaxy morphologies and central colors of distant galaxies together with the observed lower metallicities (Liang et al. 2004) and overall higher star-formation rates at high $z$ are the ingredients for an updated scenario of bulge and disk formation in spirals. Hammer et al. (2005) propose three different phases of galaxy evolution: the mergers/interacting, the compact galaxy and finally the growth of disk phase. During the last 8 Gyrs, most luminous galaxies are expected to experience a major merger that suppresses the disk as matter is falling to the mass barycenter. This phase is associated with short (1 Gyr) and strong peaks of star formations. Most of galaxies in this phase are LIRGs. Then, the compact phase corresponds to a decrease over 0.6-2 Gyr of the enhanced star formation due to merging. A significant fraction of stars form in bulges and additional occurrence of gas infall may subsequently wrap 
around the bulge to form a new disk-like component. Finally, the star formation spreads over all the forming disk as fed by large amounts of gas infall. In this scenario, about half of the bulge stellar content was made earlier in their progenitors, before the last major phase of accretion. More than a third of the present-day stellar mass is formed at $z<1$. This scenario is in very good agreement with the hydrodynamical numerical simulations of Scannapieco \& Tissera (2003) in which mergers, through secular evolution and fusions, transform galaxies along the Hubble sequence by driving a morphological loop that might also depend on the properties of the central potential wells, which are also affected by mergers. This very attractive scenario links in a simple way the distant and local galaxies; it will be confronted to the new panchromatic studies of Spitzer galaxies. Note that another possibility of buildup of dense central component in disk galaxies is internal secular evolution, as reviewed by Kormendy \& Kennicutt (2004).

\section{GALAXIES AT REDSHIFTS $\mathrm{Z} \geq 1.5$}

Analysis of the CIB in the light of the ISO observations shows that, as we go to wavelengths much longer than the emission peak, the CIB should be dominated by galaxies at higher redshifts as illustrated in Figure 4. The comoving infrared production rate needed to fill the CIB around $1 \mathrm{~mm}$ at a redshift centered around 2.5 to 3 remains comparable to the one from galaxies detected in the ISOCAM surveys and filling $65 \%$ of the peak of the CIB. In this section we discuss the rapidly growing observational evidence that this picture is basically correct. The main source of these observations has been the SCUBA submillimeter observations at $850 \mu \mathrm{m}$ and $450 \mu \mathrm{m}$ (see Blain et al 2002 for a review) and observations from the MAMBO instrument on the IRAM 30-m telescope at 1.2 mm (Greve et al. 2004). The negative Kcorrection becomes very effective at these wavelengths, leading to an almost constant observed flux for galaxies of the same total infrared luminosity between redshifts 1 and 5. More recently, the Spitzer observatory has produced a wealth of early observations showing that this observatory will contribute much to our understanding of infrared galaxies at $\mathrm{z} \geq 1.5$.

\subsection{Number Counts, Contribution to the CIB}

Blank-field deep surveys combined with mapping of areas lensed by clusters lead to number counts at $850 \mu \mathrm{m}$ down to $0.5 \mathrm{mJy}$ (e.g., Smail et al. 2002; Wang et al. 2004). At $1.2 \mathrm{~mm}$ counts have been obtained down to 2.5 mJy (e.g., Greve et al. 2004). The number counts shapes at $850 \mu \mathrm{m}$ and $1.2 \mathrm{~mm}$ are compatible with the assumption that they are made of the same population with a flux ratio $F_{850} / F_{1200}=2.5$. For a typical ULIRG SED, a $5 \mathrm{mJy}$ source at $850 \mu \mathrm{m}$ has a luminosity of $10^{12} L \odot$ at a redshift of about 2.5. The large fraction of the background resolved at $850 \mu \mathrm{m}$ (see Section 3.2) has interesting consequences. It shows very directly that if the sources are at redshift larger than 1 (as confirmed by the redshift surveys discussed below), the infrared luminosity of the sources that dominate the background is larger than $10^{12} L_{\odot}$. This is a population with a very different infrared luminosity function than the local or even the $z=1 \mathrm{lu}$ minosity function. The link between this population at high $z$, and what has been seen at $z \sim 1$ (as discussed in Section 4) will be done by Spitzer/MIPS observations at $24 \mu \mathrm{m}$. Figure 4 shows that the building on the bulk of the CIB near its peak (at $150 \mu \mathrm{m}$ ) with redshift is expected to be similar to the building of the $24 \mu \mathrm{m}$ background when the history of the 15 and $70 \mu \mathrm{m}$ CIB have larger contributions from redshift-1 sources. The K-correction plots (Figure 6]) show for $15 \mu \mathrm{m}$ a hump at $z=1$ associated with the coincidence of the 6-9 $\mu \mathrm{m}$ aromatic features in the ISOCAM filter and a hump at the same redshift for the $24 \mu \mathrm{m}$ MIPS filter associated with the 11-14 $\mu \mathrm{m}$ set of aromatic features in the MIPS filter. For the MIPS filter a second hump is visible at $z \sim 2$ that corresponds to $6-9 \mu \mathrm{m}$ features centered on the $24 \mu \mathrm{m}$ MIPS filter. ISOCAM galaxies contribute to about $2 / 3$ of the energy peak of the CIB. Following the previous considerations, it is easy to understand why the remaining fraction is likely to be made of sources in the redshift range 1.5-2.5. The presently detected SMGs with luminosity $10^{12} L_{\odot}$ have an almost constant flux between redshift 1.7 and redshift 2.5 at $24 \mu \mathrm{m}$ (similar to the constant flux at $850 \mu \mathrm{m}$ between redshift 1 and 5). The MIPS $24 \mu \mathrm{m}$ deep surveys (e.g., Papovich et al. 2004) 


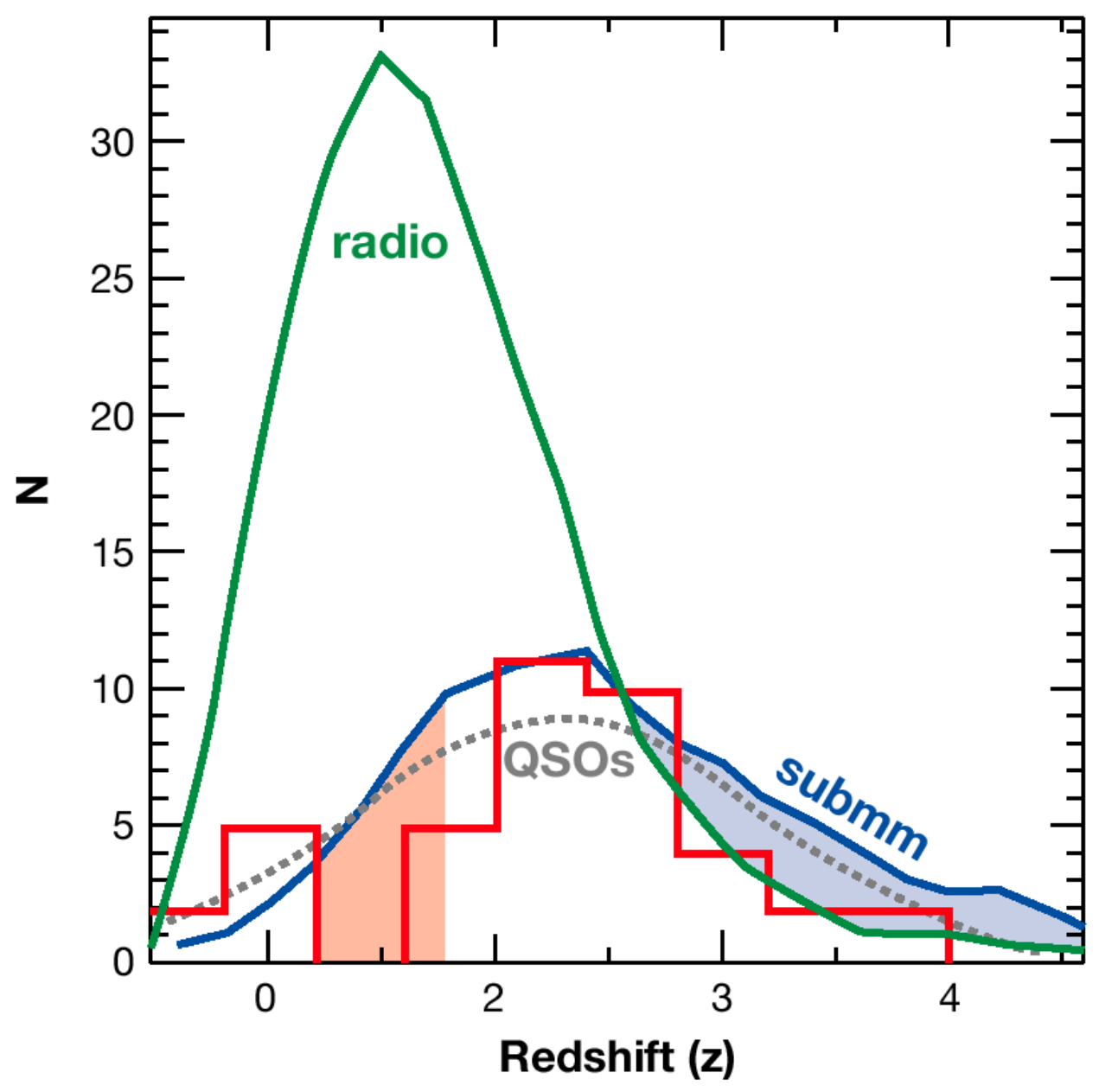

FIG. 7.- The observed histogram of the redshift distribution for the 55 radio-identified SCUBA galaxies (red histogram). Curves derived for a model of the radio/submillimeter galaxy populations (Chapman et al. 2003b; Lewis et al. 2005) are overplotted suggesting that the redshifts of the sources missed in the radio identification process lie mostly at redshifts $\sim 3-5$ between the radio and submillimeter model tracks. A sample of radio-selected QSOs is also overplotted (gray dotted line), revealing a remarkable similarity with the observed distribution for submillimeter galaxies. From Chapman et al. 2003a.

reach a sensitivity of $50 \mu \mathrm{Jy}$ and thus can detect all these galaxies when they are starburstdominated. Considering the speed of the MIPS it is likely that $24 \mu \mathrm{m}$ surveys will become the most efficient way to search for luminous starburst galaxies up to $z=2.5$ and up to 3 for the most luminous ones.

\subsection{Redshift Distribution and SEDs of the SMGs}

The first obvious question when investigating the nature of the submillimeter galaxies (SMGs) is their redshift distribution. The rather low angular resolution of the submillime- ter and millimeter observations made identifications with distant optical galaxies an almost impossible task without an intermediate identification. This is provided by radio sources observed with the VLA with 10 times better angular resolution. The tight correlation between far-infrared luminosity and radio flux (Helou et al. 1985; Condon 1992) provides the needed link. This then allows us to get optical identifications and redshift measurements using 10-m class telescopes. Confirmation of these identifications can then be obtained through CO line observations with the millimeter interferome- 
ters such as the Plateau de Bure interferometer. The redshift deduced from the optical lines is confirmed by the CO observations. So far, only a handful of cases have gone through this whole chain of observations (e.g., Genzel et al. 2003; Greve et al. 2005; Neri et al. 2003), but a high success rate gives confidence in the first step of the identification process. The chain will also have to be applied to the tentative counterparts of radio-undetected SMGs that have been found using a certain combination of optical properties (Pope et al. 2005).

The difficulty of making large, blind surveys at $850 \mu \mathrm{m}$ at the required sensitivity has lead to an attempt to find distant SMGs through blind surveys at different wavelengths. Barger et al. (2000) have observed optically faint radio sources at submillimeter wavelength and demonstrated them to be, so far, the most efficient way to preselect targets for submillimeter observations and to get larger samples of potentially high redshift SMGs. As an example, Chapman et al. (2002) recovered at $850 \mu \mathrm{m} 70 \%$ of the blind submillimeter survey sources. This contrasts the recovery rate of MAMBO sources, which is relatively low, $\sim 25 \%$ (Dannerbauer et al. 2004). It should be noticed that the radio preselection biases the sample against very high redshifts $(z<3)$ because the radio flux at $1.4 \mathrm{GHz}$ is below the detection limit of the VLA surveys used for this preselection. A model by Chapman et al. (2003b) and by Lewis et al. (2005) illustrate this effect very well (Figure 7). A fraction of the submillimeter-selected sources are missed in such a process at $z>$ 3 (detectability in radio) and around $z \sim 1.5$ (optical redshifts desert). The number of non identified submillimeter sources (around 30\% for $S_{850}>3 \mathrm{mJy}$ ) is consistent with this model. Nevertheless the submillimeter-selected sources do not appear qualitatively different from the optically-faint-radio selected ones. Another bias is the effect of the dust effective temperature of the SMGs (Lewis et al. 2005). At a given total far-infrared luminosity, hotter sources have lower submillimeter fluxes if the radio/far-infrared correlation continues to hold. They could be missed in the submillimeter surveys (see the discussion in Chapman et al. 2005).
Chapman et al. (2003a) got spectroscopic redshifts of 55 sources obtained in this way. The redshift distribution for these sources is shown in Figure 7 (note that when this review was being edited, Chapman et al. (2005) publish spectroscopic redshifts for $73 \mathrm{SMGs)}$. This distribution peaks at $\mathrm{z}=2.4$ with a substantial tail up to $\mathrm{z}=4$. In fact the redshift distribution can be represented by a Gaussian distribution centered on 2.4 and with a sigma of 0.65 . Almost all SMGs are found in the redshift range $1.5<z<3$. This redshift distribution is compared with that of the redshift distribution for a pure radio sample in Figure 7. The SMGs selected in the way described above is also shown to be very similar to the redshift distribution of the radio-selected QSOs. This observation is interesting in the context of high rate of AGN activity detected in SMGs.

The determination of the SED of millimeter/submillimeter galaxies remains an open question despite a lot of work in the last few years. The SCUBA and MAMBO data provide constraints on the flux and spectrum at long wavelengths; Spitzer observations constrain the near and mid-infrared. The far-infrared part of the SED remains the least precisely known. Low angular resolution makes 70 and $160 \mu \mathrm{m}$ deep surveys confusion-limited at 3 and $40 \mathrm{mJy}$ (Dole et al. 2004b). These limits are too high to complete the SED of the SMGs (see Figure 9). Stacking sources will help to go deeper than the confusion limit when large samples of SMGs are available in MIPS cosmological surveys. A first attempt on a radio-selected sample lowered the limit down to $1.2 \mathrm{mJy}$ at $70 \mu \mathrm{m}$ (Frayer et al. 2004). They find a typical flux ratio $\mathrm{I}(70) / \mathrm{I}(24)<7$ that they interpret to be low when compared with low-redshift starburst. However, such low ratios are typical of dusty starbursts placed at redshift greater than 1.5 . It is thus likely that the lower colors are due to a redshift effect. Appleton et al. (2004) looked at the mid- and far-infrared fluxes from a purely radio-selected $1.4 \mathrm{GHz} \mu \mathrm{Jy}$ sample of about 500 and 230 sources at 24 and $70 \mu \mathrm{m}$, respectively. They show that the far-infrared to radio correlation that is constant out to $z=1$ seems to be constant using $24 \mu \mathrm{m}$ out to $z=2$ but with a 
larger dispersion due systematic variations in SED shape throughout the population. This provides positive evidence of the universality of the infrared/radio correlations out to redshifts of about 2 .

Blain et al (2004a) have analyzed SEDs of infrared galaxies assuming that the low-redshift radio/far-infrared correlation applies to SMGs. Under this reasonable assumption and using a model of long-wavelengths SEDs based on a single modified black body, they can choose a single parameter to built an SED that fits the longwavelengths data and the radio/infrared luminosity ratio. In their paper, this single parameter is the temperature, but it could equally well be the long-wavelength emissivity, because they showed that this is degenerate with temperature. A split between two redshift populations appear in their analysis. The high- $z$ galaxies selected by the submillimeter observations are significantly colder that the low- $z$ galaxies (Dune \& Eales, 2001; Stanford et al. 2000), IRAS or IRAS-radio selected. The discrepancy in part probably reflects selection effects in the way these samples were obtained and may reflect the fact that SMGs and local infrared galaxies are distinct populations. It remains an open question what effect this has on the SED model. The main worry is that a single modified black body often does not fit ULIRGs SED when they are known at many frequencies. The SED is broader; the unavoidable temperature distribution of dust in infrared galaxies would affect such an analysis. In fact, the Stanford et al. (2000) sample does not agree well with the single-temperature SED, and this led Lagache et al. (2004) to take broader SEDs for their starburst galaxy templates.

\subsection{Nature of the SMGs}

Many LIRGs and ULIRGs at low redshifts have been identified with interacting or galaxy mergers. A substantial fraction show signs of AGN activity but it has been shown for the low-redshift LIRGs and ULIRGs that the starburst component dominates the energy output (Genzel et al. 1998; Lutz et al. 1998). The sources used for the redshift distribution by Chapman et al. (2003a) have been imaged with the HST. Most of them are multi-component-distorted galaxy systems
(Conselice et al. 2003; Smail et al. 2004). They display irregular and frequently highly complex morphologies compared to optically selected galaxies at similar redshifts. They are often red galaxies with bluer companions, as expected for interacting, star-forming galaxies. They have higher concentrations, and more prevalent major-merger configurations than opticallyselected galaxies at $z \sim 2-3$. Most strikingly, most of the SMGs are extraordinarily large and elongated relative to the field population regardless of optical magnitude (Chapman et al. 2003c). SMGs have large bolometric luminosities, $\sim 10^{12}-10^{13} \mathrm{~L}_{\odot}$, characteristic of ULIRGs. If the far-infrared emission arises from the star formation, the large luminosities translate to very high SFR $\geq 1000 \mathrm{M}_{\odot}$ year ${ }^{-1}$. Such high rates are sufficient to form the stellar population of a massive elliptical galaxy in only a few dynamical times, given a sufficient gas reservoir. SMGs are very massive systems with typical mass of $1-2 \times 10^{11} \mathrm{~L}_{\odot}$ (Swinbank et al. 2004), comparable to the dynamical mass estimates from $\mathrm{CO}$ observations. Genzel et al. (2004; and more recently Greve et al. 2005) have undertaken an ambitious program to study the nature of the SMGs in more details. They got CO spectra with the Plateau de Bure interferometer for 7 sources out of their sample of 12 for the CO 3-2 and 4-3 transitions redshifted in the $3 \mathrm{~mm}$ atmospheric window. They provide optical identifications and redshifts. The detection of these sources at the proper redshift confirms the usefulness of identification with the help of the radio sources. The median redshift of this sample is 2.4. In addition, one source was studied with the SPIFI instrument on the ESO/VLT. These observations are giving very interesting clues on the nature of the submillimeter galaxies. The gas masses obtained for these systems using CO luminosity/mass of gas determined from local ULIRGs is very large with a median of $2.2 \times 10^{10} M_{\odot}$ (10 times larger than in the Milky Way). Using the velocity dispersion, they could infer that the dynamical median mass of these systems is 13 times larger than in Lyman-break galaxies (LBGs) at the same redshift or 5 times the mass of optically selected galaxies at this redshift. These SMGs with a flux at $850 \mu \mathrm{m}$ larger than $5 \mathrm{mJy}$ are not very rare 


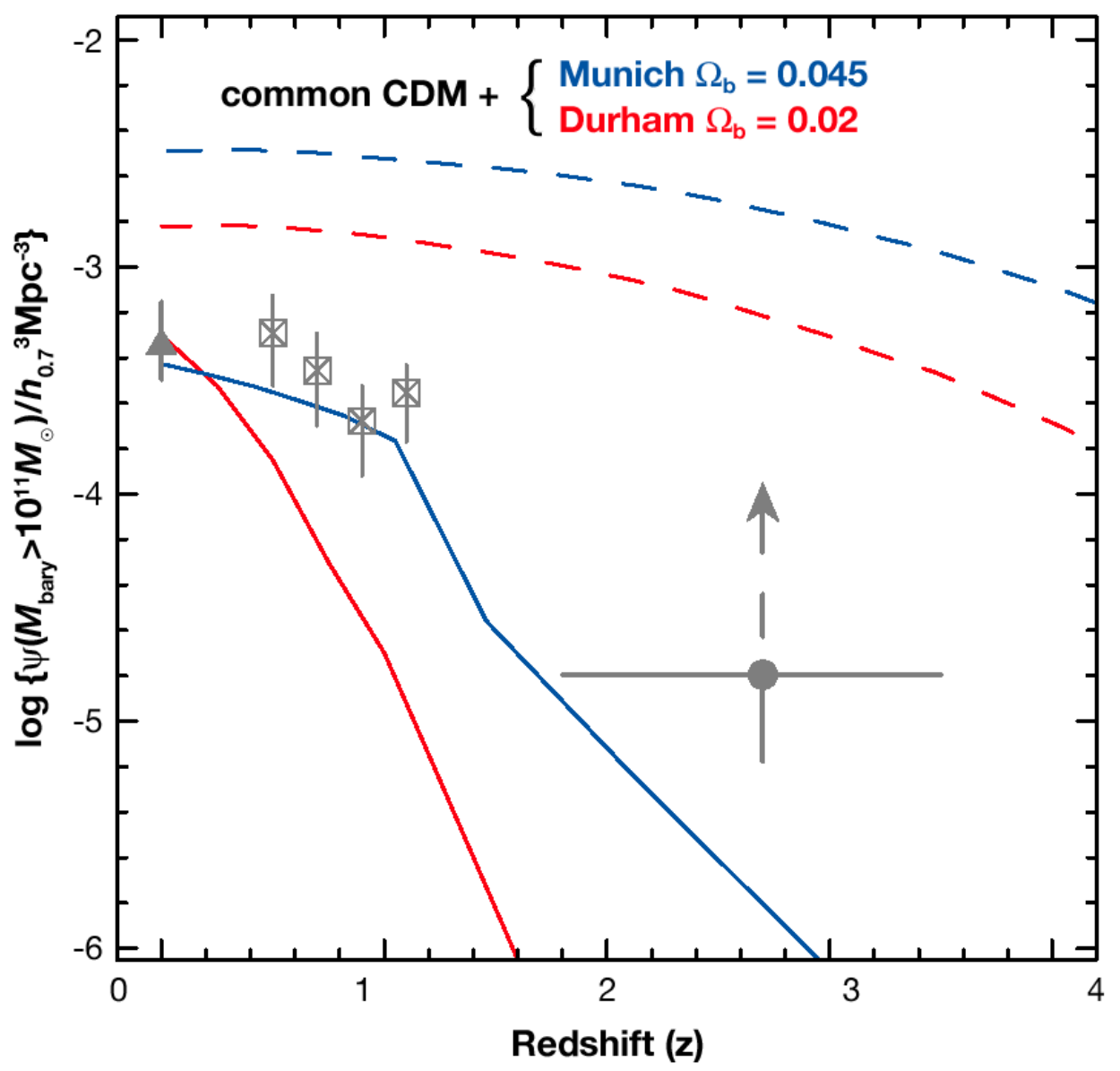

FIG. 8.- Comoving number densities of galaxies with baryonic masses $\geq 10^{11} M_{\odot}$ as a function of redshift. The triangle and open squares show densities of massive stellar systems at $z=0$ and $z \sim 1$; The circle shows the density for massive SMGs at $z \sim 2.7$, with a factor of 7 correction for burst lifetime. Blue and red curves show the predictions of semianalytic models by the "Munich" and "Durham" groups, respectively. Dashed curves show the corresponding number densities of halos with available baryonic masses $\geq 10^{11} M_{\odot}$. The two models use the same halo simulations but assume different $\Omega_{\mathrm{b}}$. From Genzel et al. (2004).

and unusual objects, because they contribute to about $20 \%$ of the CIB at this frequency. Through multiwavelength observations, Genzel et al. (2004) get the stellar component in $\mathrm{K}$ band, and infer the star-formation rate and duration of the star-formation burst. They can then compare the number density of these massive systems with semiempirical models of galaxy formation. The very interesting result is that this number density is significantly larger than the predicted one, although the absolute numbers depends on a number of assumptions like the IMF. The comparison is shown in Figure 8 . Such massive systems at high redshift are not easy to understand in current cold dark matter hierarchical merger cosmogonies. However, one must keep in mind that bright SMGs $\left(\mathrm{S}_{850}>5 \mathrm{mJy}\right)$ that contribute $20 \%$ of the CIB may not be representative of the whole population. Gravitational lens magnification provides a rare opportunity to probe the nature of the distant sub-mJy SMGs. Kneib et al. (2005) study the property of one SMG with an $850 \mu \mathrm{m}$ flux $\mathrm{S}_{850}=0.8 \mathrm{mJy}$ at a redshift of $z=2.5$. This galaxy is much less luminous and massive than other high- $z$ SMGs. It resembles to similarly luminous dusty starbursts resulting from lower-mass mergers in the local Universe.

In order to link the different population of 
high-redshift objects, several LBGs at redshift between 2.5 and 4.5 have been targeted at $850 \mu \mathrm{m}$. The Lyman-break technique (Steidel et al. 1996) detects the rest-frame $91.2 \mathrm{~nm}$ neutral hydrogen absorption break in the SED of a galaxy as it passes through several broad-band filters. LBGs are the largest sample of spectroscopically confirmed high-redshift galaxies. Observing LBGs in the submillimeter is an important goal, because it would investigate the link, if any, between the two populations. However, the rather low success rate of submillimeter counterpart of LBGs (e.g., Chapman et al. 2000; Webb et al. 2003) argues against a large overlap of the two populations.

\subsection{Spitzer $24 \mu m$ Sources}

A potential new way to find high- $z$ LIRGs and ULIRGs appeared recently with the launch of the Spitzer observatory. Particularly suited to this goal is the $24 \mu \mathrm{m}$ channel of the MIPS instrument. The confusion levels in the 70 and $160 \mu \mathrm{m}$ prevent detection a significant number of high-redshift objects, and the IRAC 3.6 to $8 \mu \mathrm{m}$ at high redshift probes mostly the old stellar component that is much weaker than the dust emission in starburst galaxies. At the time of writing, the observations are under way, and only a few results are available. Le Floc'h et al. (2004) give the first hint on the $24 \mu \mathrm{m}$ selected galaxies. They couple deep $24 \mu \mathrm{m}$ observations in the Lockman hole and extended groth strip with optical and near-infrared data to get both identification and redshift (either spectroscopic or photometric). They find a clear class of galaxies with redshift $1 \leq z \leq 2.5$ and with luminosities greater than $\sim 5 \times 10^{11} \mathrm{~L}_{\odot}$ (see also Lonsdale et al. 2004). These galaxies are rather red and massive with $\mathrm{M}>2 \times 10^{10} \mathrm{M}_{\odot}$ (Caputi et al. 2005). Massive star-forming galaxies revealed at $2 \leq z \leq 3$ by the $24 \mu \mathrm{m}$ deep surveys are characterized by very high star formation rates $-\mathrm{SFR} \geq 500 \mathrm{M}_{\odot}$ year $^{-1}$. They are able to construct a mass of $\simeq 10^{11} \mathrm{M}_{\odot}$ in a burst lifetime $(\simeq 0.1$ Gyr). The $24 \mu \mathrm{m}$ galaxy population also comprises sources with intermediate luminosities $\left(10^{10} \leq L_{I R} \leq 10^{11}\right.$ $\left.\mathrm{L}_{\odot}\right)$ and low to intermediate assembled stellar masses $\left(10^{9} \leq \mathrm{M} \leq 10^{11} \mathrm{M}_{\odot}\right)$ at $z \leq 0.8$. At low redshifts, however, massive galaxies are also present, but appear to be building their stars quiescently in long timescales (Caputi et al. 2005). At these redshifts, the efficiency of the burst-like mode is limited to low mass $\mathrm{M} \leq 10^{10} \mathrm{M}_{\odot}$ galaxies. These results support a scenario where star-formation activity is differential with assembled stellar mass and redshift, and proceed very efficiently in massive galaxies (Caputi et al. 2005).

In the Lockman Hole, only one galaxy is associated with an X-ray source. This suggests that these galaxies are mostly dominating by star formation, consistent with the findings of Alonso-Herrero et al.(2004) and Caputi et al. (2005). This is also suggested by SEDs that are best fitted by PAH features rather than by strongly rising, AGN-type continua (Elbaz et al. 2005). The selected sources exhibit a rather wide range of MIPS to IRAC flux ratio and optical/near-infrared shapes, suggesting a possibly large diversity in the properties of infrared galaxies at high redshift as noticed by Yan et al. (2004b). Based on these first analyzes, together with the interpretation of the number counts (e.g., Lagache et al. 2004 ), it is clear that the $24 \mu \mathrm{m}$ observations will provide the sample to unambiguously characterize the infrared galaxies up to $z \simeq 2.5$. They should fill the gap between the ISO- and SCUBA-selected galaxies.

Several $24 \mu \mathrm{m}$ observations have been conducted on selected ERO and SCUBA and MAMBO samples. To our knowledge, LBGs have not been observed at long wavelengths. The MAMBO/SCUBA selected galaxies in the Lockman hole with radio identification have been observed by Spitzer and most of them detected between 3.6 and $24 \mu \mathrm{m}$. This allows to get an average SED for these (Egami et al. 2004; Ivison et al. 2004; see Figure 9) Spitzer deep surveys at $24 \mu \mathrm{m}$ and shallow surveys like the SWIRE legacy (Lonsdale et al. 2004) can easily detect them and are thus a promising new way to find this class of high- $z$ infrared galaxy. Nevertheless, the Early Release Observations from Spitzer have been used to extract their submillimeter flux from a stacking analysis of SCUBA observations in the Lockman hole (Serjeant et al. 2004). In this field, seven SMGs were already known and others were identified by further analysis. For the bulk of the $24 \mu \mathrm{m}$ sources a marginal 


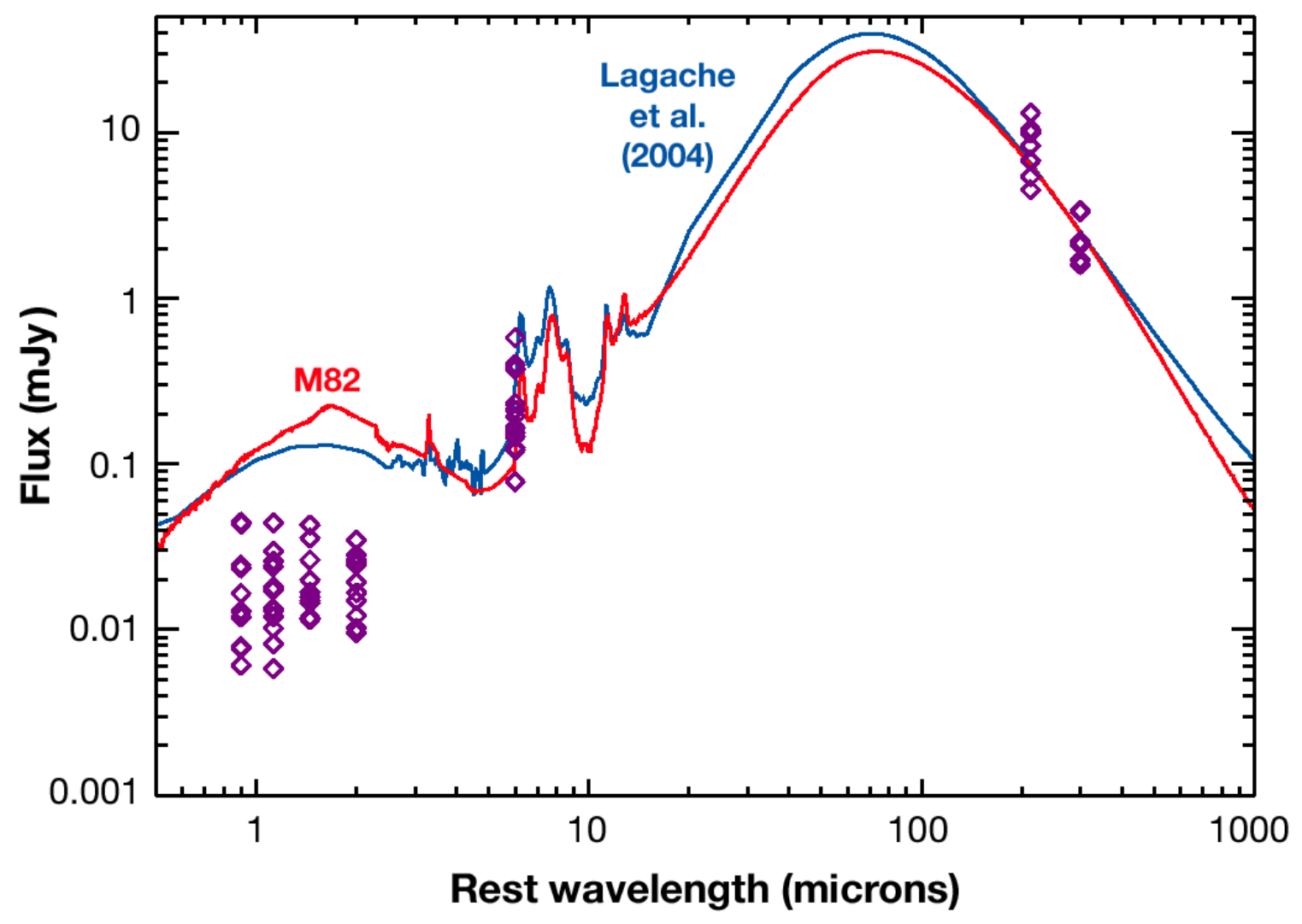

FIG. 9.- Rest-frame SED of 15 SMGs (assuming a redshift of 3) with MAMBO and/or SCUBA, Spitzer/IRAC and Spitzer/MIPS $24 \mu \mathrm{m}$ measurements. Purple diamonds are the galaxies 208, 119, 115, 48, 44 (Frayer et al. 2004), LE850_4, LE850_35 (Egami et al. 2004), and MMJ105201, MMJ105155, MMJ105203, MMJ105216, MMJ105148, MMJ105157, MMJ105207 MMJ105203 (Ivison et al. 2004). Overplotted are the SEDs of M82, normalized at $850 \mu \mathrm{m}$ (from Chanial 2003), and the SED template of the Lagache et al. (2004) model, for $\mathrm{L}=10^{13} \mathrm{~L}_{\odot}$ and a redshift of 3 (no normalization has been applied). Note that this sample of SMGs has a ratio dust/stellar component higher than the template or M82.

detection is found with an $S_{850} / S_{24}$ ratio $(1 / 20)$ much lower than that observed for SMGs. This clearly shows that the SMGs are only a fraction of the $24 \mu \mathrm{m}$ sources, as expected. An interesting challenge is to find if Spitzer color criteria can be found to extract preferentially SMGs, i.e., the galaxies that account for most of the CIB near $1 \mathrm{~mm}$. The SED in the thermal infrared appears quite variable for LIRGs and ULIRGs making this difficult (e.g., Armus et al. 2004).

Extremely Red Objects (EROs) are usually selected based on their red colors: $(R-$ $\left.K_{s}\right) \geq 5.3 \mathrm{mag}$ or $\left(I-K_{s}\right) \geq 4 \mathrm{mag}$. This color selection should include early-type galaxies at $z \sim 1$. However, the color selections are also sen- sitive to dust-reddened, star-forming systems. Up to now, it remains unclear what fraction of EROs are truly dust-obscured galaxies. Different scenarios of galaxy formation predict very different formation epochs for such galaxies. It is thus interesting to characterize these galaxies, in particular whether they belong to the early-type or dusty star-forming class of objects. Spitzer/MIPS $24 \mu \mathrm{m}$ observations offer the first opportunity to address this issue because $24 \mu \mathrm{m}$ observations can clearly discriminate between the two populations. In the N1 field, Yan et al. (2004a) suggest that about 50\% of EROs are infrared luminous, dusty starbursts at $z \geq 1$ (in a similar study, Wilson et al. (2004) show that at least $11 \%$ of $0.6<z<1.3$ EROs and at least $22 \%$ of $z>1.3$ EROs are dusty 
star-forming galaxies). Their mean $24 \mu \mathrm{m}$ flux corresponds to infrared luminosities of about $3 \times 10^{11}$ and $10^{12} \mathrm{~L}_{\odot}$ at $\mathrm{z} \sim 1$ and $z \sim 1.5$, respectively. They are massive galaxies with lower limit $\mathrm{M} \geq 5 \times 10^{9}$ to $210^{10} \mathrm{M}_{\odot}$. The fraction of EROs likely to be AGN is small; about $15 \%$. The link between the two classes of EROs could be that starburst EROs are experiencing, at $z>1$, violent transformations to become massive early-type galaxies.

\subsection{ULIRGs and Active Galactic Nuclei at High Redshifts}

SMGs are massive ULIRGs at high redshift. One of the key question discussed above for the $z \simeq 1$ galaxies is to distinguish whether starburst or AGN activity powers the dust heating and associated infrared emission. The presence of an AGN in galaxies can be investigated using optical/near-infrared, emission line diagnostics and/or X-ray observations. But the identification of the presence of an AGN does not mean that it is the dominant source of the farinfrared emission. Alexander et al. (2003; see also Almaini et al. 2003) use Chandra observations of the $\mathrm{CDF}-\mathrm{N}$ to constrain the $\mathrm{X}$-ray properties of 10 bright SMGs. Half of the sample has flat X-ray spectral slopes and luminous $\mathrm{X}$-ray emission, suggesting obscured AGN activity. However, a comparison of the AGNclassified sources to the well-studied, heavily obscured AGN NGC 6240 suggests that the AGN contributes on average a negligible fraction (about $1.4 \%$ ) of the submillimeter emission. For the MAMBO sources, similar results are found: only one out of the nine MAMBO sources studied by Ivison et al. (2004) has an X-ray counterpart. It has, as expected from low redshift ULIRGs observations (e.g., Rigopoulou et al. 1999), a different mid-infrared SED than the starburst dominated sources. About $75 \%$ of their sample has rest-frame mid-infrared to far-infrared SED commensurate with obscured starburst. Swinbank et al. (2004), using AGN indicators provided by near-infrared spectra, estimate that AGNs are present in at least $40 \%$ of the galaxies in their sample of 30 SMGs. Emission-line diagnostics suggest that star formation is the dominant power source. However, the composite spectrum for the galaxies that individually show no signs of an AGN in their near-infrared spectra appears to show an under- lying broad $\mathrm{H}_{\alpha}$ line. This suggests that even these galaxies may host a low-luminosity AGN that is undetectable in the individual spectra. All these studies tend to show that starburst activity is the dominant source of power of dust emission in the far-infrared. Still, it is rather difficult to estimate the true "contamination" by the AGN. To go deeper, Chapman et al. (2004) tried an original approach. They observe a sample of identified SMGs at high angular resolution in the radio and use the radio emission as a proxy for the far-infrared emission. This assumption is based on the wellknown very tight far-infrared/radio correlation mentioned above. If detected, an extended radio (and thus far-infrared) component is likely to arise from the star formation. The detection of extended emission requires sub-arcsec resolution to map emission on kpc-scales. These are accessible by radio interferometry (they are well beyond far-infrared and submillimeter facilities capabilities). They find that for $70 \%$ of the SMG sample, the MERLIN/VLA radio exhibits resolved radio emission which mirrors the general form of the rest frame UV morphology seen by HST. The galaxies are extended on scales of about $10 \mathrm{kpc}$. They interpret this as a strong support for the hypothesis that radio emission traces spatially extended massive star formation within these galaxies. This is clearly different from what is seen in local ULIRGs where the far-infrared/radio emission is concentrated in the compact nuclear region with an extend less than $1 \mathrm{kpc}$. In the remaining $30 \%$ of the SMG sample, the radio emission is more compact (essentially unresolved). This is a signature of either a compact nuclear starburst and/or an AGN.

In conclusion, the exact fraction of distant submillimeter and millimeter galaxies containing an energetically dominant AGN is difficult to extract from observations. However, even in the systems containing an unambiguously powerfully AGN, the far-infrared emission seems to be powered by the star formation. Surprisingly, this seems to be also the case in distant QSOs. Recently, Beelen (2004) has shown that the far-infrared and blue luminosities from the host galaxies of distant radio-quiet QSOs, are slightly correlated. The far-infrared and ra- 


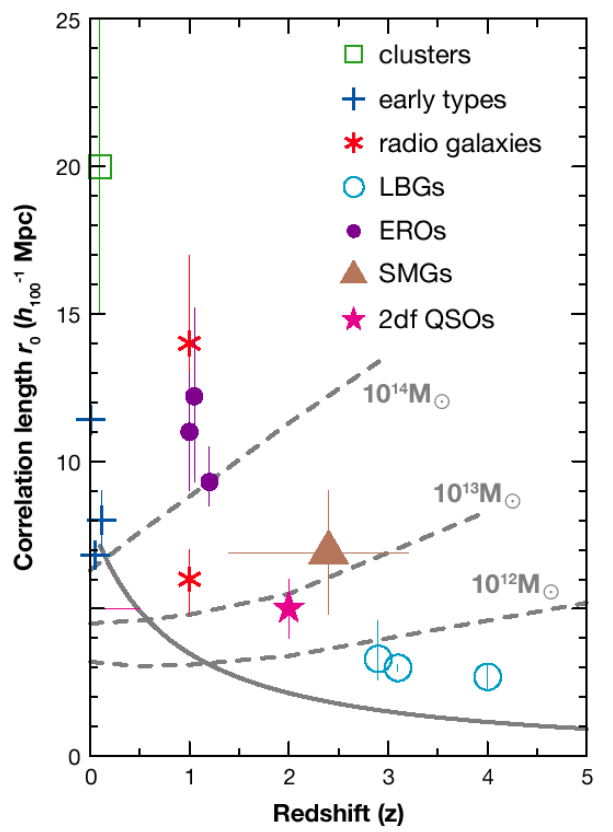

FIG. 10.- Comoving correlation length of the SMGs (triangle) in contrast to other populations of low- and high-redshift galaxies (see the summary in Overzier et al. 2003). The horizontal error bar on the SMG point spans the range of redshifts over which SMG associations are found. The solid line shows a representative model for the evolution of a certain overdensity. The dashed lines show the expected correlation length of dark matter halos as a function of mass and redshift. From Blain et al. (2004a).

dio emission of these quasars follow the radioinfrared correlation observed in local ULIRGs (Yun et al. 2001), providing a first indication that the dust is predominantly heated by the star-formation activity rather than by the AGN. Moreover, the non-linearity between the far-infrared and blue luminosities is also an indication that the heating mechanism of the dust is not directly linked to the AGN. However, the presence of this correlation could suggest a causal connection between the formation of stars in the host galaxy and the activity of the central super massive black hole. This connection has been successfully modeled by Granato et al. (2004).

Finally Houck et al. (2005) and Yan et al. (2005) demonstrate the potential of using midinfrared spectroscopy, especially the aromatic and silicate features produced by dust grains to directly probe distant $\mathrm{L} \sim 10^{13} \mathrm{~L}_{\odot}$ ULIRGs at $z \sim 2$. Spitzer/IRS observations provide a unique and direct access to high- $z$ ULIRG physical properties. It will definitively open the route toward a complete census of the distant infrared-luminous Universe. A first study on two distant SMGs using Spitzer/IRS by Lutz et al. (2005) finds for one SMG an equal contribution from star formation and AGN. The second galaxy is dominated by star formation.

\section{CLUSTERING}

Measuring clustering gives information about the distribution of galaxies with respect to the dark matter. The strength of clustering is correlated with the mass of extended halos that host luminous galaxies. At redshift lower than 1.3, large-scale structures have been mapped by the DEEP2, 2dF and SDSS surveys (Coil et al. 2004; Peacock et al. 2001; Doroshkevich et al. 2004). At higher redshifts, correlation lengths $r_{0}$ have been measured for different galaxy populations such as the LBGs and EROs. Values of about $r_{0}=4 \mathrm{~h}^{-1} \mathrm{Mpc}$ (Porciani \& Giavalisco 2002) and $r_{0}=11 \mathrm{~h}^{-1} \mathrm{Mpc}$ (Daddi et al. 2000; Firth et al. 2002; Roche et al. 2003) are reported with mean redshift of about 3 and 1-1.5 for the LBGs and EROs, respectively. Getting information on the clustering of the infrared galaxies is essential to see how they relate to the other galaxy populations and to understand their formation process. As an example, one of the key questions is to see if the most massive SMGs are associated with the most massive dark matter halo.

Up to now, very little information is available on the clustering of infrared galaxies. A clustering signal can tentatively be measured, although the small number of objects in the deep narrow-pencil ISOCAM and SCUBA beams prevents accurate measurements of the autocorrelation function. At $z \sim 1$, Moy \& Elbaz (2005) study the large-scale and close environment of ISOCAM galaxies. They find that infrared galaxies are more strongly clustered than optical galaxies. Eighty percents of ISOCAM galaxies are found preferentially in redshift peaks, versus $68 \%$ for the optically selected galaxies. Moy \& Elbaz (2005) find indirect evidences that the triggering mechanism of dusty starbursts is small-scale ( $100 \mathrm{kpc})$ galaxygalaxy interactions. Such interactions do not lead to major mergers most of the time but are 
more likely simple fly-by, tidal interactions or minor mergers. At much higher redshift, there are some indirect evidences of strong clustering of SMGs when compared to other classes of high-redshift galaxies. For example, Chapman et al. (2001) identified SMGs within the most overdense structure of LBGs at $z \sim 3.1$ (Steidel et al. 2000). De Breuck et al. (2004) detected an overdensity of MAMBO sources likely at $z \simeq 4.1$ in a proto-cluster containing also overdensities of Ly $\alpha$ emitters and LBGs. The first three-dimensional quantitative measurement of the clustering strength of SMGs has been made by Blain et al. (2004a). They find spectroscopic evidence for clustering. Using 73 spectroscopically identified galaxies, they find a surprisingly large number of "associations" with redshifts separated by less than $1200 \mathrm{~km}$ $\mathrm{s}^{-1}$. They provide tentative evidence for strong clustering of SMGs at $z \simeq 2-3$ with a correlation length of $\sim(6.9 \pm 2.1) \mathrm{h}^{-1} \mathrm{Mpc}$ using a simple pair-counting approach appropriate for the small and sparse SMG sample. This correlation length appears to be somewhat larger than that for both LBG and QSO galaxies at comparable redshifts. It is thus unlikely that the SMGs form a simple evolutionary sequence with either population. On the contrary, the correlation length could be consistent with a form of evolution that subsequently matches the large comoving correlation length typical of evolved EROs at $z \simeq 1$ and of clusters of galaxies at $z=0$ (Figure 10). From this figure, we see that the correlation function of SMGs appears to be consistent with the hypothesis that they are associated with the most massive dark matter halos at high redshifts. These are more massive than the host halos of LBGs and QSOs at comparable redshift. These preliminary conclusions have to be investigated in more detail. In particular, the SMG masses inferred from clustering measurements have to be compared to the dynamical masses derived from millimeter wave $\mathrm{CO}$ spectroscopy. To go deeper in understanding the clustering properties of infrared galaxies, Spitzer $24 \mu \mathrm{m}$ surveys will be best suited. With a large number of sources detected in quite large surveys, Spitzer will unambiguously constrain the clustering of infrared galaxies from redshift 0.5 to 2.5. Conjointly, the physics of galaxy clustering can be probed by the CIB fluctuation analysis (Knox et al. 2001). CIB fluctuations measure, on large angular scales, the linear clustering bias in dark-matter halo and, at small angular scales, the nonlinear clustering within a darkmatter halo (Cooray \& Sheth 2002). They thus probe both the dark-matter halo mass scale and the physics governing the formation of infrared galaxies within a halo. Promising attempts are underway using the $170 \mu \mathrm{m}$ ISO FIRBACK fields (Lagache et al., in preparation).

\section{FINDING ULIRGS AT $Z>3$ : CONFUSION}

In the near future, when a proper census of ULIRGs up to $z \simeq 3$ will have been carried out, the fraction of the CIB at $\sim 1 \mathrm{~mm}$ not accounted for should give an indication of the contribution from sources at larger redshifts. Deep surveys at $\sim 1-2 \mathrm{~mm}$ are the only obvious tool to find most of these sources. However, the limiting factor of the surveys is not only detector sensitivity or photon noise but also confusion. We concentrate in this section only on extragalactic sources confusion. Source confusion in the far-infrared is illustrated in Figure 11

Predicting or measuring confusion depends on the scientific goal of the measurement (Helou \& Beichman 1990; Dole et al. 2003; Lagache et al. 2003). Performing an unbiased far-infrared or submillimeter survey and getting a complete sample has different requirements than following-up in the far-infrared an already known near-infrared source to get an SED and/or a photometric redshift. In the former case, one has to tightly control the statistical properties of the whole sample; in the latter case, completeness is irrelevant, and even a low photometric accuracy is adequate. We thus favor the use of a term like "unbiased confusion" for the former case. New techniques are being developed to use a priori information at shorter wavelength (e.g., $8 \mu \mathrm{m}$ with Spitzer/IRAC and $24 \mu \mathrm{m}$ with MIPS) to infer some statistical properties (such as source density or SED) of sources at longer wavelength (e.g., 24 or $160 \mu \mathrm{m}$, respectively), and thus to beat unbiased confusion. Predicting unbiased confusion (for instance Condon 1974; Franceschini et al. 1989; Helou \& Beichman 1990; Dole et al. 2003; Lagache et al. 2003; Takeuchi et 


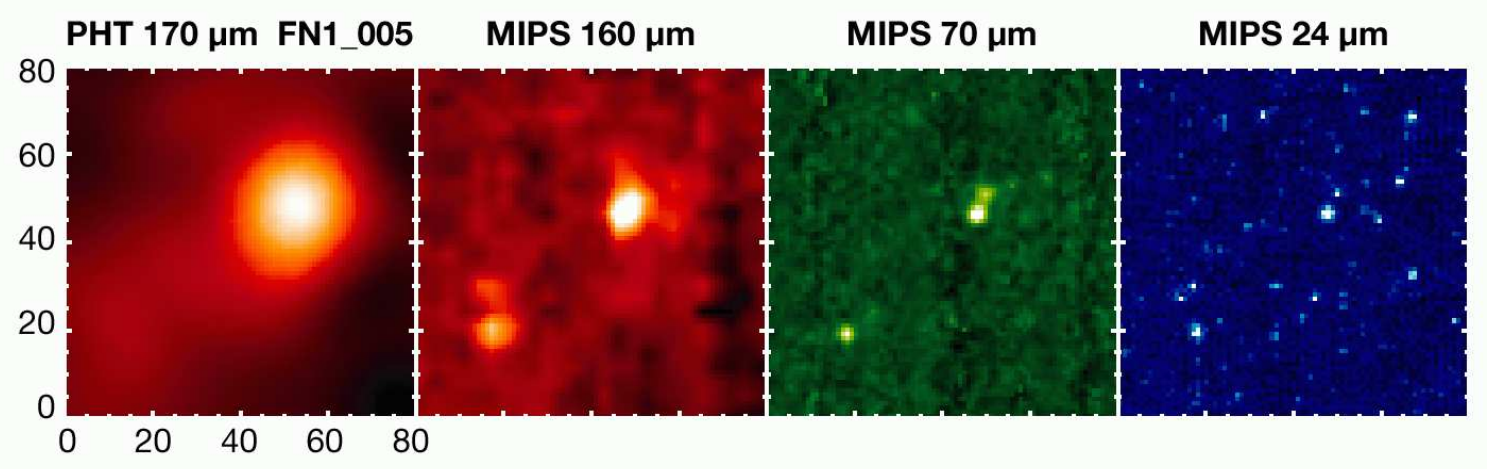

FIG. 11. - Effects of confusion in the far-infrared. Observation of a source in the ELAIS-N1/FIRBACK field in a 400x400 square arcsec box. All images have been resampled to 5 arcsec per pixel, which oversamples the far-infrared maps but undersamples the mid-infrared map. From left to right: $373 \mathrm{mJy}$ ISOPHOT $170 \mu \mathrm{m}$ source with about 128s of integration (FIRBACK survey, Dole et al. 2001); labels indicate the 5 arcsec pixels; Spitzer/MIPS $160 \mu \mathrm{m}$ with about 16s of integration (SWIRE survey, Lonsdale et al. 2004); MIPS $70 \mu \mathrm{m}$ with about 80s of integration (SWIRE); MIPS $24 \mu \mathrm{m}$ with about 160s of integration (SWIRE). Notice (1) the ISO $170 \mu \mathrm{m}$ source is marginally resolved with MIPS 160, and is unambiguously resolved at $70 \mu \mathrm{m}$ and $24 \mu \mathrm{m} ;(2)$ the two fainter MIPS $160 \mu \mathrm{m}$ resolved sources (bottom left) create fluctuations in the ISO $170 \mu \mathrm{m}$ map that produce the confusion noise when the resolution is limited.

al. 2004; Negrello et al. 2004) requires the knowledge of at least the number-count distribution of the galaxies. In practice, models (validated at some point by observations) are used. Because the shape of the counts in a $\log (N)-\log \left(S_{\nu}\right)$ diagram varies with the flux density $S_{\nu}$, the fluctuation level of faint sources below $S_{\nu}$ also vary. This fluctuation level gives an estimate of the unbiased confusion using a photometric criterion (Lagache \& Puget 2000; Dole et al. 2003; Lagache et al. 2003). At very faint fluxes, when the background is almost resolved, the photometric criterion will obviously give a very small value for the unbiased confusion level, but the observations will be limited by the confusion due to the high density of faint resolved sources. Thus, another criterion, the source density criterion for unbiased confusion (SDC, Dole et al. 2003; Dole et al. $2004 \mathrm{~b}$ ), needs to be computed and compared to the photometric criterion.

In the infrared and submillimeter range below $300 \mu \mathrm{m}$, the unbiased confusion is in general better predicted by the source density criterion for current and future facilities, because the angular resolution has improved (e.g. from ISO to Spitzer). At longer wavelengths, the photometric criterion is more useful. We use the model of Lagache et al. (2004) to predict unbiased confusion. Fifteen-meter submillime- ter telescopes are limited by the confusion at $1.2 \mathrm{mJy}$ at $850 \mu \mathrm{m}$ and at $0.5 \mathrm{mJy}$ at $1.2 \mathrm{~mm}$. Ongoing surveys (Smail et al. 2002; Greve et al. 2004) already reach or are about to reach these levels. If we want to resolve about $80 \%$ of the CIB, which corresponds to $56 \mu \mathrm{Jy}$ at $850 \mu \mathrm{m}$ and $20 \mu \mathrm{Jy}$ at $1.3 \mathrm{~mm}$, one would need a $\sim 90$ $\mathrm{m}$ telescope at $850 \mu \mathrm{m}$ and a $\sim 150$-m telescope at $1.4 \mathrm{~mm}$. Future facilities for infrared and submillimeter observations include far-infrared space observatories such as Herschel, SPICA, and SAFIR, survey missions like ASTRO-F and Planck, a larger near-infrared and mid-infrared observatory, JWST, and a ground-based submillimeter interferometer, ALMA. In order to detect LIRGs at $z \sim 3$, experiments operating at $15,24,70,160,450,850,1380 \mu \mathrm{m}$ respectively should reach a sensitivity of $1,1,8,50$, 200, 70, $30 \mu \mathrm{Jy}$, respectively (see Figure [12). The spectral window around $450 \mu \mathrm{m}$ seems the most effective to reach these galaxies. This constraint is somewhat relaxed if one wants to detect ULIRGs at $z \sim 3$, in which case the required sensitivities are multiplied by about 10 . In the near future, ASTRO-F, Herschel/SPIRE and Planck will be mostly limited by confusion. At long wavelengths, to probe most of the CIB source population and to detect enough early mergers made by building blocks not yet affected by star formation and evolution, large 


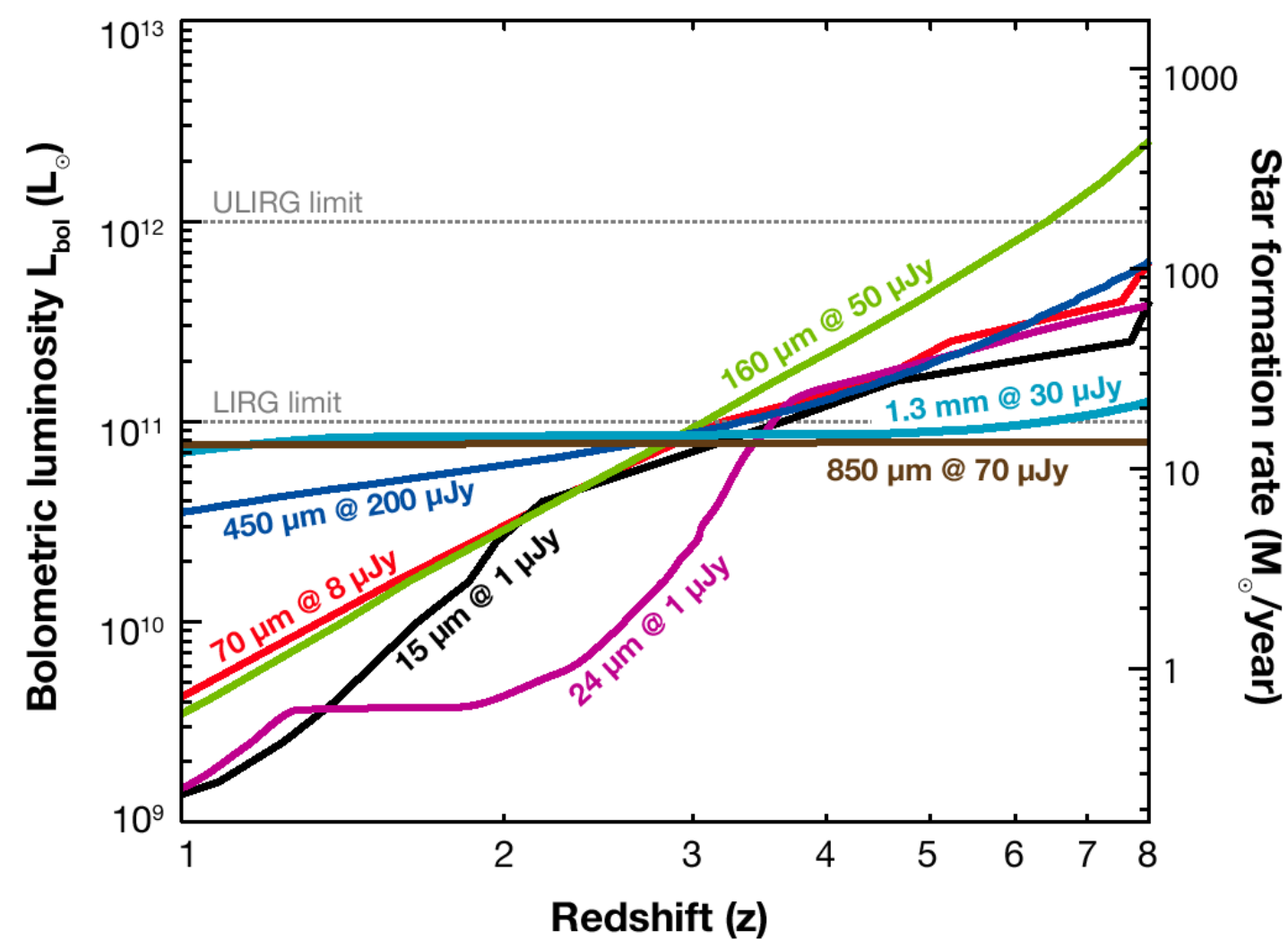

FIG. 12.- Sensitivity to the bolometric luminosity (and star-formation rate, assuming star-forming galaxies) of hypothetical surveys designed to detect LIRGs at $z \sim 3$. The required sensitivities are: at $15 \mu \mathrm{m}: S_{\nu}>1 \mu \mathrm{Jy}$; at $24 \mu \mathrm{m}: S_{\nu}>1 \mu \mathrm{Jy}$; at $70 \mu \mathrm{m}: S_{\nu}>8 \mu \mathrm{Jy} ;$ at $160 \mu \mathrm{m}: S_{\nu}>50 \mu \mathrm{Jy} ;$ at $450 \mu \mathrm{m}: S_{\nu}>200 \mu \mathrm{Jy} ;$ at $850 \mu \mathrm{m}: S_{\nu}>70 \mu \mathrm{Jy}$; at $1.3 \mathrm{~mm}: S_{\nu}>30 \mu \mathrm{Jy}$. This plot makes use of the Lagache et al. (2004) model (see appendix).

extragalactic surveys will have to be conducted with ALMA. These surveys will take a substantial fraction of the time (Lagache et al. 2003). As an example, mapping one square degree at $1.3 \mathrm{~mm}$ at the $5 \sigma$ sensitivity of $1 \mathrm{mJy}-\sim 50 \%$ of the CIB is resolved - takes 138 days without including overheads.

\section{COMPARISON OF MODELS WITH OBSERVATIONS}

One of the striking result of the deep surveys concerns the evolution of the infrared and submillimeter galaxy population. The source counts are high when compared to no evolution, or moderate, evolution models ${ }^{1}$ for infrared galaxies. Classical semianalytical models of galaxy formation predicts neither the large

\footnotetext{
1 'No-evolution': the co-moving luminosity function remains equal to the local one at all redshifts
}

numbers of infrared galaxies nor their very strong evolution, revealing a serious gap in our understanding of galaxy formation and evolution. Very recently, several empirical approaches have been proposed to model the high evolution of the infrared output with redshift (e.g., Chary \& Elbaz 2001; Franceschini et al. 2001; Rowan-Robinson 2001; Takeuchi et al. 2001; Xu et al. 2001; Lagache et al. 2004) that fit source counts, redshift distributions and CIB intensities and fluctuations, although often not all of them. All these models, however, agree on a general trend - i.e., the luminosity function must change dramatically with redshift, with a rapid evolution of the high-luminosity sources $\left(\mathrm{L}>2 \quad 10^{11} \mathrm{~L}_{\odot}\right)$ from $z=0$ to $z=1$, which then stay rather constant up to redshift 3 or more. The evolution of the infrared luminosity function may be linked to a 
bimodal star-formation process, one associated with the quiescent and passive phase of the galaxy evolution and one associated with the starburst phase, triggered by merging and interactions. The latter dominates the infrared and submillimeter energy density of the Universe at high $z$. Consistently, cold dark matter N-body simulations show that halo merger rates increase with redshift as $(1+\mathrm{z})^{\mathrm{m}}$ with $2.5 \leq \mathrm{m} \leq 3.5$ (Gottlober et al. 2001). Observations, however, give $m$ values between 0 and 4 (Le Fèvre et al. 2000; Conselice et al. 2003; Bundy et al. 2004; Lin et al. 2004). The spread is due to different selection effects, detection techniques, pair criteria and sample variance. It is therefore not easy to reconcile the different observational results. Moreover, comparisons with models are very difficult because definitions of merger rates may not be consistent. Merger rates can also depend on halo masses. As a consequence, the timescale of the merger phase is difficult to estimate. Peaks of star formation produced by mergers in hydrodynamical models (e.g., Scannapieco \& Tissera 2003) has a duration of several hundreds of million years. This is consistent with what is observed. ULIRGs emit more than half of their bolometric luminosity from a starburst of age $10^{7}-10^{8}$ years (Genzel et al. 1998). LIRGs build up their stellar mass in a typical timescale of about 0.1 Gyr (Franceschini et al. 2003). These timescales are also supported by Marcillac et al. (2005) who performed Monte Carlo simulations using synthetic spectra based on the models of Bruzual and Charlot (2004) to derive the past star-formation history of 22 LIRGs. They found that LIRGs experience a major event of star formation in their lifetime that produce about $10 \%$ of their stellar mass within 0.1 Gyr. How many such episodes of violent star formation does a typical galaxy experience? Assuming a timescale of $0.1 \mathrm{Gyr}$, Hammer et al. (2005) estimate the number of episodes per galaxies as about 5 from $z=1$ to $z=0.4$. These episodic bursts naturally explain the high fraction of LIRGs in the distant Universe.

Models that are more sophisticated than empirical approaches attempt to follow the physics of galaxy formation in greater detail (e.g.,
Guiderdoni et al. 1998; Hatton et al. 2003; Granato et al. 2004; Silva et al. 2005). In semianalytical models, the collapse of perturbations is described by the classical top-hat model under the assumptions of homogeneity and sphericity. The mass distribution of collapsed halos is computed from the so-called peaks formalism developed by Bardeen et al. (1986). Then dissipative collapse and cooling are introduced, with the usual "overcooling" problem that can partly be solved by introducing stellar feedback. Star-formation processes are deduced from the gas content and the dynamical timescale of the galaxies. Finally spectrophotometric evolution is used to compute the age dependence of the gas content, the spectra of the stellar populations and the mass-toluminosity ratios. To make specific predictions for the infrared galaxies, these models must include an important additional feature: absorption of the UV/optical radiation and emission by the dust grains. Very often two modes of star formations are considered; a quiescent mode and a burst mode in which the star formation timescales are much shorter. This burst mode is triggered by galaxy mergers and is absolutely required by the infrared to submillimeter observations. There are some indications that to reproduce the submillimeter galaxy counts, a dramatic change of the IMF is required. A topheavy IMF, in particular, increases the production of dust that is essential for boosting the luminosity of galaxies in the submillimeter. Using an IMF of the form $d N / \operatorname{dnn} m \propto m^{-x}$ with $x=0$ for the burst mode, Baugh et al. (2005) were able to reproduce not only the submillimeter observations but also the properties of LymanBreak galaxies. They predict that the SMGs reside in the more massive halos in place at $z=2$ and therefore that they are more strongly clustered than dark matter at this epoch. This is consistent with tentative observational constraints (Blain et al. 2004a). There are several observational "indications" of massive stars (> $100 \mathrm{M}_{\odot}$ ) in nearby starburst templates. WolfRayet stars ${ }^{2}$ have been detected in a large num-

\footnotetext{
2 Wolf-Rayet stars are hot $(25,000$ to $50,000 \mathrm{~K})$, massive $\left(\geq 25 M_{\odot}\right)$, luminous stars with a high rate of mass loss. The Wolf-Rayet phase appears in an advanced stage of evolution. They are believed to be O stars that have lost their hydrogen envelopes, leading their helium cores exposed. Wolf-Rayet stars are often in a binary
} 
ber of galaxies undergoing intense bursts of star formation (e.g., Gonzalez-Delgado et al. 1997; Pindao et al. 2002). However, it remains difficult to measure the IMF at high mass because of aging effects that can mimic real upper-mass IMF cutoff (the highest massive stars have very short lifetimes).

In conclusion, the hierarchical galaxy formation paradigm is very successful in its description of large-scale structure formation and evolution. The next important step will be to test this picture to explain not only the number densities but also the mass assembly and particularly the mass of the SMGs. First mass measurements of SMGs galaxies seem to show that a very flat IMF cannot by itself explain the mass assembly of the baryonic matter at high $z$ (Genzel et al. 2004). Hierarchical clustering underpredicts the high- $z$ volume densities of these massive galaxies. More work needs to be done to test the baryonic mass assembly in the hierarchical paradigm. Both observational and model estimates are still very uncertain, with the former depending on large lifetime corrections and small samples and the latter on ad hoc input recipes for feedback and star formation.

\section{CONCLUSION AND OPEN QUESTIONS}

A number of conclusions are now clear from the analysis of the identified sources in the CIB:

- The comoving energy produced in the past that makes up the CIB at different wavelengths is more uniform that what is suggested by its spectral energy distribution. This is due to the fact that the CIB at long wavelengths $(\lambda \geq 400 \mu \mathrm{m})$ is dominated by emission from the peak of the SED of galaxies at high $z$. More quantitatively, the ISOCAM surveys reveal that about two-thirds of the CIB emission at $\lambda \sim 150 \mu \mathrm{m}$ is generated by LIRGs at $z \sim 0.7$. At $850 \mu \mathrm{m}$, more than half of the submillimeter CIB is generated by SMGs. The brightest SMGs $\left(S_{850}>3 \mathrm{mJy}, \sim 30 \%\right.$ of the CIB) are ULIRGs at a median redshift of 2.2. The energy density at $150 \mu \mathrm{m}$, which is $\sim 20-25$ times larger than the energy density at $850 \mu \mathrm{m}$ requires a comoving energy production rate at $z=0.7$

system, and are deemed, within a few million years, to explode as type Ib or Ia supernovae. roughly 10 times the energy production rate at $z=2.2$.

- The evolution exhibited by LIRGs and ULIRGs is much faster than for optically selected galaxies. The ratio of infrared to optical, volume-averaged output of galaxies increases rapidly with increasing redshift.

- Luminosity function evolution is such that the power output is dominated by LIRGs at $z \simeq 0.7$ and ULIRGs at $z \simeq 2.5$.

- The energy output of CIB sources is dominated by starburst activity.

- AGN activity is very common in the most luminous of these galaxies even though this activity does not dominate the energy output. The rate and fraction of the energy produced increase with the luminosity.

- LIRGs at $z \simeq 0.7$ are dominated by interacting massive late-type galaxies. Major mergers become dominant in ULIRGs at $z \simeq 2.5$.

- SMGs show rather strong correlations with correlation lengths larger than those of other high redshift sources.

- LIRGs and ULIRGs cannot be identified with any of the distant populations found by rest-frame ultraviolet and optical surveys.

Although these findings are answering the basic questions about the sources that make up the CIB, there are still observational difficulties to be overcome to complete these answers. The SEDs of LIRGs and ULIRGs are quite variable and often not very well constrained in their ratio of far-infrared to mid-infrared or to submillimeter wavelengths. The far-infrared, where most of the energy is radiated, requires cryogenically cooled telescopes. These have small diameters and, hence, poor angular resolution and severe confusion limits for blind surveys. Establishing proper SEDs for the different classes of infrared galaxies detected either in mid-infrared (with ISOCAM at $15 \mu \mathrm{m}$ or MIPS at $24 \mu \mathrm{m}$ ) or in millimeter-submillimeter surveys is one of the challenges of the coming decade. Making 
sure that no class of sources that contribute significantly to the CIB at any wavelength has been missed is an other observational challenge. The submillimeter galaxies not found through the radio-selected sources and the question of the warm submillimeter galaxies are also two of those challenges.

Multiwavelength observations of high- $z$ infrared galaxies give a number of new insights on the galaxy formation and evolution problem. As an example, the gas masses and total masses of SMGs are found to be very high. There is a first indication that the number of such high-mass object at redshifts between 2 and 3 is uncomfortably large compared to semianalytical models of galaxy formation based on the standard hierarchical structure-formation frame. The evolution of the luminosity function is dominated by more luminous sources as redshift increases. This is surprising because the mass function of the collapsed structure is expected to be dominated by smaller and smaller objects as redshift increases.

The populations of infrared galaxies concentrated at $z \simeq 1$ and at $z \simeq 2.5$ studied so far reveal rather different type of sources. The lower redshift ones seem to be starburst phases of already-built massive, late-type field galaxies accreting gas or gas-rich companions forming the disks. We see today a rapid decrease of this activity probably associated with a dry out of the gas reservoir in their vicinity. The larger redshift ones, which are also more luminous, seem to belong to more massive complex systems involving major merging. These systems could be located in the rare larger amplitude peaks of the large-scale structures leading to massive elliptical galaxies at the center of rich clusters. The redshift distribution of these seems quite similar to the redshift distribution of quasars.

Interesting problems that are central to the understanding of galaxy formation and evolution have to be solved in the next decade:

- Determining the role of the large scale environment (nodes, filaments and sheets of the large-scale structures) on star formation;

- Find the relative rates of accretion of gas and smaller galaxies in the growth of massive objects;
- Establish the cycle of bulge versus disk formation, as a function of the ratio between stars and gas in the accreted material;

- Identify the different types of starburst (in a disk or in the nucleus, interaction or merger driven); and

- Estimate the fraction of time spent in the starburst phase and the duration of this phase

Current observations all point in the direction of a possible strong effect of the large-scale environment and the need for models of hierarchical formation and evolution that include properly star versus gas ratio in the accreted material.

Finally the connection between the starburst phenomenon and the AGN activity is an old question still largely unresolved. Recent observations of infrared/submillimeter galaxies have reinforced the link but have not much improved our understanding of the physical link.

\section{ACKNOWLEDGMENTS}

We are very grateful to Alexandre Beelen, Karina Caputi, David Elbaz, François Hammer, and George Helou for very useful discussions during the writing of this manuscript. We also thank Pierre Chanial for providing us the M82 SED. Finally, we warmly thank the scientific editor, who found a large number of typos and mistakes in our use of English. The reading of the paper has been significantly improved by his detailed corrections.

\section{APPENDIX: THE PHENOMENOLOG- ICAL MODEL OF LAGACHE ET AL. (2004)}

In this paper, we make extensive use of the Lagache et al. (2004) phenomenological model to illustrate our points. This model constrains in a simple way the evolution of the infrared luminosity function with redshift. It fits all the existing source counts consistent with the redshift distribution, the CIB intensity, and, for the first time, the CIB fluctuation observations, from the mid-infrared to the submillimeter range. In this model, Lagache et al. (2004) assume that 
infrared galaxies are mostly powered by star formation and hence they use SEDs typical of starforming galaxies. Although some of the galaxies will have AGN-dominated SEDs, they are a small enough fraction that they do not affect the results significantly. They therefore construct "normal" and starburst galaxy template SEDs: a single form of SED is associated with each activity type and luminosity. They assume that the luminosity function is represented by these two activity types and that they evolve independently. They search for the form of evolution that best reproduces the existing data. An example of two cosmological implications of this model is $(a)$ the PAH features remain prominent in the redshift band $0.5-2.5$ (as observa- tionally shown by e.g., Caputi et al. 2005), and (b) the infrared energy output has to be dominated by $\sim 3 \sim 10^{11} \mathrm{~L}_{\odot}$ to $\sim 3 \times 10^{12} \mathrm{~L} \odot$ galaxies from redshift 0.5 to 2.5 .

The excellent agreement between the model and all the available observational constraints makes this model a likely good representation of the average luminosity function as a function of redshift and a useful tool to discuss observations and models. Its rather simple assumptions such as the single parameter sequence of SEDs for starburst galaxies is certainly not accounting for some of the detailed recent observations but probably do not affect seriously the redshift evolution of the averaged properties which are what is modeled.

\section{REFERENCES}

Alexander D.M., Bauer F.E., Brandt, W.N.et al. 2003, AJ 125,383

Almaini O., Scott S.E., Dunlop J.S. et al. 2003, MNRAS 338,303

Alonso-Herrero A., Pérez-gonzález P.G., Rigby J. et al. 2004, APJS 154, 155

Appleton P.N., Fadda D.T., Marleau F.R., et al. 2004, APJS 154, 147

Armand C., Milliard B., Deharveng J. M. 1994, A\&A, 284, 12

Armus L., Charmandaris V., Spoon H.W.W. et al. 2004, ApJS 154, 178

Aussel H., Cesarsky C.J., Elbaz D., Starck J.-L. 1999, A\& A 342, 313

Bardeen J.M., Bond J.R., Kaiser N., Szalay A.S. 1986, ApJ 304, 15

Barger A.J., Cowie L. L., Richards E.A. 2000, AJ 119, 2092

Baugh C.M., Lacey C.G., Frenk C.S. et al. 2005, MNRAS 356, 1191

Beelen A., 2004. PhD Thesis. Paris-XI Univ.

Bell E.F., Papovich C., Wolf C., et al. 2005, ApJ 625, 23

Bernstein R.A., Freedman W.L., Madore B.F. 2002, ApJ 571, 56

Blain A.W., Chapman S.C., Smail I., Ivison R.J. 2004a, ApJ 611, 725

Blain A.W., Chapman S.C., Smail I., Ivison R. 2004b, ApJ 611, 52

Blain A.W., Smail I., Ivison R.J. et al. 2002, Physics Report 369, 111

Bruzual G., Charlot S. 2003 MNRAS 344, 1000

Bundy K., Fukugita M., Ellis R.S. et al. 2004, ApJ 601, L123

Calzetti D., Kinney A.L., Storchi-Bergmann T. 1994, ApJ 429, 582

Cambrésy L, Reach W.T., Beichman C.A., Jarrett T.H. 2001, ApJ 555, 563

Chanial P., 2003. PhD Thesis. Paris-XI Univ.

Chapman S.C., Blain A.W, Ivison R.J., Smail I., 2003a. Star formation through time, Granada Spain, Oct.2002. ASP Conf. Ser. 289

Chapman S.C., Blain A.W, Smail I., Ivison R.J., 2005, ApJ 622, 772

Chapman S.C., Helou G., Lewis G., Dale, D., 2003b, ApJ 588, 186
Chapman S.C., Lewis G.F., Scott D. et al., 2002 ApJ 570,557

Chapman S.C., Lewis G.F., Scott D. et al., 2001, ApJ 548,17

Chapman S.C., Scott D., Steidel C.C., et al., 2000, MNRAS 319, 318

Chapman S.C., Smail I., Windhorst R. et al., 2004, ApJ 611,732

Chapman S.C., Windhorst R., Odewahn S., et al., 2003c, ApJ 599, 92

Chary R., Elbaz D. 2001, ApJ 556, 562

Coil A.L., Davis M., Madgwick D.S., et al. 2004, ApJ 609,425

Condon J.J. 1974, ApJ 188, 279

Condon J. J. 1992, ARAA 30, 575

Conselice C.J., Chapman S.C., Windhorst R.A. 2003, ApJ 596, 5

Cooray C.J., Sheth R. 2002, Phys. Rep. 372, 1

Daddi E., Cimatti A., Pozzetti L. et al. 2000, A\&SA 361, 535

Dannerbauer H., Lehnert M.D., Lutz D. et al. 2004, ApJ 606, 664

De Breuck C., Bertoldi F., Carilli et al. 2004, A\&A 424, 1

Désert F.-X., Boulanger F., Puget J.-L. 1990, A\&GA 237, 215

Dole H., Gispert R., Lagache G. et al. 2001, A\& A 372, 364

Dole H., Lagache G., Puget J-L., 2003 ApJ 585, 617

Dole H., Le Floc'h E., Pérez-González P.G. et al., 2004a, ApJS 154, 87

Dole H., Rieke G.H., Lagache G. et al., 2004b, ApJS 154, 93

Doroshkevich A., Tucker D. L., Allam S., Way M.J. 2004, A\&A 418, 7

Dunne L., Eales S.A. 2001, MNRAS 327, 697

Dwek E., Krennrich F. 2005, ApJ 618, 657

Egami E., Dole H., Huang J.-S. et al. 2004, ApJS 154, 130

Elbaz D., Césarsky C. 2003, Science 300, 270

Elbaz D., Césarsky C.J., Chanial P. et al. 2002, $A \mathscr{E} A$ 384,848

Elbaz D., Césarsky C., Fadda D., et al. 1999, A\& $A$ 351, L37

Elbaz D., Le Floc'h E., Dole H., Marcillac D. 2005, A\&A 434,1 
Fadda D., Elbaz D., Duc P.-A. et al. 2002, A\&A 361, 827

Filipenko A.V. 1992, Relat. Between Act. Galact. Nucl. Starburst Galaxies: ASP Conf. Ser., 31, 485

Firth A.E., Somerville R.S., McMahon R.G. et al. 2002, MNRAS 332, 617

Flores H., Hammer F., Elbaz D. et al. 2004, A\&A 415, 885

Flores H., Hammer F., Thuan T.X., et al. 1999, ApJ 517,148

Franceschini A., Aussel H., Césarsky C.J. et al. 2001, A\&A 378, 1

Franceschini A., Berta S., Rigopoulou D. et al. 2003, $A \& A 403,501$

Franceschini A., Toffolatti L., Danese L., De Zotti G. 1989, ApJ, 344, 35

Frayer D.T., Chapman S.C., Yan L. et al., 2004, ApJS 154,137

Galliano F. 2004, PhD Thesis. Paris XI Univ.

Genzel R., Baker A.J., Ivison R.J. et al. 2004. Proc. Venice Conf. Multiwavelength Mapping of Galaxy Formation and Evolution. In press

Genzel R., Baker A.J., Tacconi L. et al. 2003, A\& A 584, 633

Genzel R., Césarsky C.J. 2000, ARAA 38, 761

Genzel R., Lutz D., Sturm E. et al. 1998, ApJ 498,579

Gispert R., Lagache G., Puget J.-L. 2000, $A \mathscr{E} A$ A 360, 1

Gonzalez-Delgado R.M., Leitherer C., Heckman T.M., Cervino M. 1997, ApJ 483, 705

Gorjian V., Wright E.L., Chary R.R. 2000, ApJ 536, 550

Gottlober S., Klypin A., Kravtsov A.V. 2001, ApJ 546, 223

Granato G.L., De Zotti G., Silva L. et al. 2004, ApJ 600, 580

Greve T. R., Bertoldi F., Smail I. et al. 2005, MNRAS 359,1165

Greve T. R., Ivison R. J., Bertoldi, F. et al. 2004, MNRAS 354, 779

Gruppioni C., Lari C., Pozzi F. et al. 2002, MNRAS 335, 831

Guiderdoni B., Hivon E., Bouchet F.R., Maffei B. 1998, MNRAS 295, 877

Guiderdoni B. \& the Galics collaboration, 2004. The dusty and molecular Universe; A prelude to Herschel and ALMA, ed. A. Wilson, ESA Conf. Ser.

Hammer F., Flores H., Elbaz D. et al. 2005, A\&A 430, 115

Hammer F., Gruel N., Thuan T.X. et al. 2001, ApJ 550, 570

Hatton S., Devriendt J.E.G., Ninin S. et al. 2003, MNRAS 343, 75

Hauser M.G., Arendt R.G., Kelsall T. et al. 1998, ApJ 508,25

Hauser M.G., Dwek E. 2001, Ann. Rev. Astron. Astrophys. 37, 249

Helou G., Beichman C.A. 1990. From Ground-Based to Space-Borne Sub-mm Astronomy. ESA SP-314, p. 117

Helou G., Lu N.Y., Werner M.W. et al. 2000, ApJ 532, 21

Helou G., Soifer B.T., Rowan-Robinson M. 1985, ApJ 298,7

Héraudeau P., Oliver S., del Burgo C. et al., 2004, MNRAS 354, 924

Houck J.R., Soifer B.T., Weedman D. et al. 2005, ApJ 622, L105

Ivison R.J., Greve T.R., Serjeant S. et al. 2004, APJS 154,124

Joseph R.D. 1999, Astrphys. Space Sci. 266, 321

Kashlinski A. 2005, Physics report 409, 361
Kawara, K., Matsuhara H., Okuda H. et al. 2004, $A \mathscr{E} A$ 413,843

Kennicutt R.C. 1992, AJ 388, 310

Kennicutt R.C. Jr. 1998, Ann. Rev. Astron. Astrophys. 36,189

Knox L., Cooray A., Eisenstein D. et al. 2001,ApJ 550,

Kormendy J., Kennicutt R.C. 2004, Ann. Rev. Astron. Astrophys. 42, 603

Kneib J.-P., Neri R., Smail I., et al. 2005, A\&SA 434, 819

Lagache G., Dole H. 2001, A\&A 372, 702

Lagache G., Dole H., Puget J.-L. et al. 2003, MNRAS 338,555

Lagache G., Dole H., Puget J.-L. et al. 2004, APJS 154, 112

Lagache G., Haffner L.M., Reynolds R.J., Tufte S.L. 2000, $A \mathscr{E} A$ A 354, 247

Lagache G., Puget J.-L. 2000, A $\& A$ 355, 17

Lari C., Pozzi F., Gruppioni C., et al. 2001 MNRAS 325 1173

Laurent O., Mirabel I.F., Charmandaris V. et al. 2000, $A \mathscr{E} A 359,887$

Le Fèvre O., Abraham R., Lilly S.J. et al. MNRAS 311, 565

Le Floc'h E., Papovich C., Dole H. et al. 2005, ApJ, in press

Le Floc'h E., Pérez-González P.G., Rieke G.H. 2004 ApJS 154, 170

Lewis G.F., Chapman S.C., Helou G. 2005, ApJ 621, 32

Li A., Draine B.T. 2001, ApJ, 554, 778

Liang Y.C., Hammer F., Flores H. et al. 2004, A\&A 423, 876

Lin L.W., Koo D.C., Willmer C.N.A. et al. 2004, ApJ 617, L9

Lonsdale C., Del Carmen Polletta M.C., Surace J. et al., ApJS 154, 54

Lutz D., Spoon H.W.W., Rigopoulou D. et al. 1998, ApJ 505,103

Lutz D., Valiente E., Sturn E. et al. 2005, ApJ 625, 83

Lutz D., Veilleux S., Genzel R. et al. 1999, ApJ 517, 13

Mann R.G., Oliver S., Carballo et al. 2002, MNRAS 332, 549

Mattila K. 2003, ApJ 591, 119

Matsumoto T., Matsuura S., Murakami H. et al. 2005, ApJ 626, 31

Marcillac D., Elbaz D., Charlot S. et al. 2005, A\&A submitted

Metcalfe L., McBreen B., Kneib J.-P. et al. 2003, $A \& A$ 407, 791

Miville-Deschenes M-A., Lagache, G., Puget J-L. 2002, A\& A 393, 749

Nakamura O., Fukugita M., Brinkmann J., Schneider D.P. 2004, AJ 127, 2511

Negrello N., Magliocchetti M., Moscardini L. et al. 2004 MNRAS 352, 493

Neri R., Genzel R., Ivison R.J. et al. 2003, ApJ 597, 113

Oliver S., Rowan-Robinson M., Alexander D.M. et al. 2000, MNRAS 316, 749

Overzier R. A., Röttgering H. J. A., Rengelink, R. B., Wilman, R. J. 2003, A\& A 405, 53

Papovich C., Dole H., Egami E., et al. 2004, ApJS 154, 70

Partridge R.B., Peebles P.J.E. 1967, ApJ 148, 377

Peacock J.A., Cole S., Norberg P. et al. 2001, Nature 410, 169

Pérez-González P.G., Rieke G.H., Egami E. et al. 2005, $A p J$. In press

Peeters E., Spoon H.W.W., Tielens A.G.G.M. 2004, ApJ 613,986 
Pindao M., Schaerer D., Gonzalez Delgado R.M., Stasinska G. 2002, A\&A 394, 443

Pope A., Borys C., Scott D. et al. 2005, MNRAS 358, 149

Porciani C., Giavalisco M. 2002, ApJ 565, 24

Puget J.-L., Abergel A., Bernard J.P. et al. 1996, $A \& A$ 308, L5

Renault C., Barrau A., Lagache G., Puget J-L. 2001, A $\&$ A 371, 771

Rigopoulou D., Spoon H.W.W., Genzel R. et al. 1999, AJ 118,2625

Rieke G.H., Lebofsky M.J. 1979, Ann. Rev. Astron. Astrophys. 17, 477

Roche N.D., Dunlop J., Almaini O. 2003, MNRAS 346, 803

Rowan-Robinson M. 2001, ApJ 549, 745

Sanders D.B., Mirabel F. 1996, ARAA 34, 749

Sanders D.B., Soifer B.T., Elias J.H. et al. 1988a, ApJ 325,74

Sanders D.B., Soifer B.T., Elias J.H. et al. 1988b, ApJ 328, L35

Sato Y., Kawara K., Cowie L.L. et al. 2003, A $\mathscr{\mho} A$ 405, 833

Scannapieco C., Tissera P.B. 2003, MNRAS 338, 880

Serjeant S., Morthier A.M.J., Ivison R.J. et al. 2004 APJS 154, 118

Silva L., De Zotti G., Granato G.L. et al. 2005, MNRAS 357,1295

Smail I., Chapman S.C., Ivison R.J., Blain A.W. 2005, ApJ 616, 71

Smail I., Ivison R.J., Blain A.W., Kneib J.-P. 2002, MNRAS 331, 495

Soifer B.T., Neugebauer G. 1991, AJ 101, 354

Solomon P.M., Vanden Bout P.A. 2005, Ann. Rev. Astron. Astrophys. 43. In press
Stanford S.A., Stern D., van Breugel W., De Breuck C. 2000, ApJS 131,185

Steidel C.C., Adelberger K.L., Shapley A.E. et al. 2000, ApJ 532, 170

Steidel C.C., Giavalisco M., Pettini M. et al. 1996, ApJ 624, L17

Swinbank A.M., Smail I., Chapman et al. 2005, ApJ 617, 64

Takeuchi T. T., Ishii T. T. 2004, ApJ 604, 40

Takeuchi T.T., Ishii T.T., Hirashita H. et al., 2001 PASJ 53, 37

Toomre A. 1997, Conf. Proc. Evol. Galaxies Stellar Popul., May 19-21, ed B.M. Tinsley, R.B. Larson, p. 401. New Haven: Yale University Observatory

Veilleux S., Kim D.-C., Sanders D.B. et al., 1995, APJS 98,171

Wang W.H., Cowie L.L., Barger A.J. 2004, ApJ 613, 655

Webb T.M., Eales S., Foucaud S. et al., 2003, ApJ 582 6

Wilson G., Huang J.-S., Pérez-González P.G. et al., 2004, ApJS 154, 107

Wright E.L. 2001, ApJ 553, 538

Xu C., Lonsdale C.J., Shupe D.L. et al. 2001, ApJ 562, 179

Yan L., Chary R., Armus L. et al. 2005, ApJ. In press

Yan L., Choi P.I., Fadda D. et al. 2004a, $A p J S$ 154, 75

Yan L., Helou G. Fadda D. et al. 2004b, ApJS 154, 60

Yun R., Naveen A., Condon J.J. 2001, ApJ 554, 803

Zheng X.Z., Hammer F., Flores H. et al. 2004, A $\& A 421$, 